\title{
Synthesis of 3,4-dihydro-2H-pyrrole 1-oxide based aldonitrones as potential spin trapping agents
}

\author{
Andrey I. Taratayko, ${ }^{\text {a,b* }}$ Christina S. Becker, ${ }^{\text {a,b }}$ Igor' A. Grigor'ev, ${ }^{\text {a }}$ Yurii V. Gatilov, ${ }^{\text {a }}$ \\ Tatyana V. Rybalova, and Vladimir A. Reznikov ${ }^{\text {a,b }}$ \\ ${ }^{a}$ N. N. Vorozhtsov Novosibirsk Institute of Organic Chemistry, Siberian Branch of the Russian \\ Academy of Sciences, Akad. Lavrent'ev Ave. 9, 630090 Novosibirsk, Russia \\ ${ }^{b}$ Novosibirsk State University, Pirogova Street 2, 630090 Novosibirsk, Russia \\ E-mail:taratayk@nioch.nsc.ru
}

\begin{abstract}
Different synthetic approaches to fluorescent 3,4-dihydro- $2 \mathrm{H}$-pyrrole 1-oxide aldonitrones that are of potential interest as spin traps are discussed.
\end{abstract}

Keywords: Aldonitrones, spin traps, 3,4-dihydro-2H-pyrrole 1-oxide, fluorescence, organometallic compounds

\section{Introduction}

Aldonitrones based on pyrroline N-oxide derivatives (type A, scheme 1) are widely used as spin traps of short-living radicals in biological systems ${ }^{1,2,3}$ that permits the determination of both the concentration and a structure of these radicals by the analysis of ESR of spin adducts of type $\mathbf{B}$ formed (scheme 1). This method possesses some obvious limitations. Different types of radicals react with nitrones with different rates, ${ }^{4}$ and spin adducts formed have different lifetime and the main route of their transformation is bio-reduction to the hydroxylamines (type $\mathbf{C}$, scheme 1). Thereby, the concentration of the spin adduct may be insufficient for the ESR spectrum registration and its interpretation. Furthermore, signals in spectra can broaden due to high solution viscosity or presence of dissolved oxygen, etc., hindering the interpretation of the spectra observed.

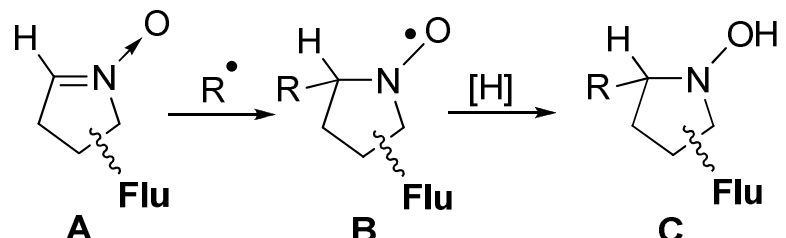

Scheme 1. The essence of spin trapping. 
It is well known that the presence of both fluorescent fragment and nitroxide group in one molecule leads to significant decrease of quantum yield of fluorescence. This phenomenon was proposed to use in the systems where transformation "nitroxide - hydroxylamine" takes place (as a result of bio-reduction) for biochemical studies. ${ }^{5}$ This phenomenon probably may be used for the detecting of short-living radicals in "inversed manner". In the case of conversion of diamagnetic spin trap into spin adduct - nitroxide the initial fluoresce quenching should be observe, that could provide the possibility of measurement of the radical concentration based on fluorescent quantum yield decrease. ${ }^{6,7}$ This approach doesn't allow the determination of the nature of free radical, but seems to be prospective to increase the sensitivity in comparison to ESR method.

Thus, the aim of this work is the synthesis of aldonitrones (ketonitrones possess lower rates of free radicals capture ${ }^{8}$ ) based on 3,4-dihydro- $2 H$-pyrrole 1-oxides containing fluorescent substituent. Noteworthy, that the presence of hydrophilic group is highly desirable in order to increase the solubility of spin trap in water phase that is very important particularly in biochemical investigations.

\section{Results and Discussion}

Different synthetic approaches were used to achieve the posed goal. They can be conditionally divided into two types. The first is the insertion of fluorescent moiety at the stage of heterocycle ring construction and the second is based on modifications of suitable heterocycle using reactivity of nitrone or other groups in a molecule.

The general method to synthesize the derivatives of 3,4-dihydro- $2 H$-pyrrole 1 -oxide is based on the condensation reaction of aliphatic nitro compounds with $\alpha, \beta$-unsaturated carbonyl compounds followed by reductive cyclization of initially formed 1,4-nitroaldehydes or 1,4nitroketones. ${ }^{9,10}$

Starting 2b, containing naphthalene-1-yl group as fluorescent moiety and ester group for further transformation into hydrophilic carboxyl group could be synthesized by reaction of ethyl 2-bromo-2-(naphthalene-1-yl)acetate with sodium nitrite in DMF in the presence of phloroglucinol similarly to that, described for ethyl 2-bromophenylacetate. ${ }^{11}$ However in the case of 1 the main, if not the sole product of the reaction was ethyl 2-hydroxy-2-(naphthalene-1yl)acetate 2a, probably due to significant steric hindrance (Scheme 2).<smiles>CCOC(O)c1cccc2ccccc12</smiles>

2a

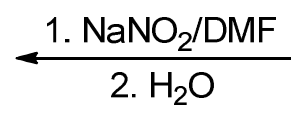

2. $\mathrm{H}_{2} \mathrm{O}$<smiles>CCOC(=O)C(Br)c1cccc2ccccc12</smiles>

1

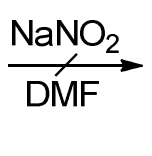<smiles>CCOC(=O)C(c1cccc2ccccc12)[N+](=O)[O-]</smiles>

2b

Scheme 2. Reaction of bromide 1 with sodium nitrite. 
An alternative approach for fluorescent nitrones synthesis could be the condensations of acinitro compounds salts bearing fluorescent fragment and cyano group as a precursor of hydrophilic moiety, with acrolein. Sodium salts $\mathbf{4 a , b}$, were synthesized by condensation of nitriles 3a,b with methyl nitrate in the presence of sodium methylate (Scheme 3). Reaction of compounds $\mathbf{4 a , b}$ with acrolein in the presence of double excess of acetic acid leads to compounds 5a,b, but complete consumption of reagents wasn't observe. Increasing of the reaction time from 24 to 48 hours under similar conditions leads to products yield decrease (from $65 \%$ to $48 \%$ for $\mathbf{5 a}$ ) (Scheme 3). The structure of $\mathbf{5 a}$ was proved by X-ray analysis (Figure 1).

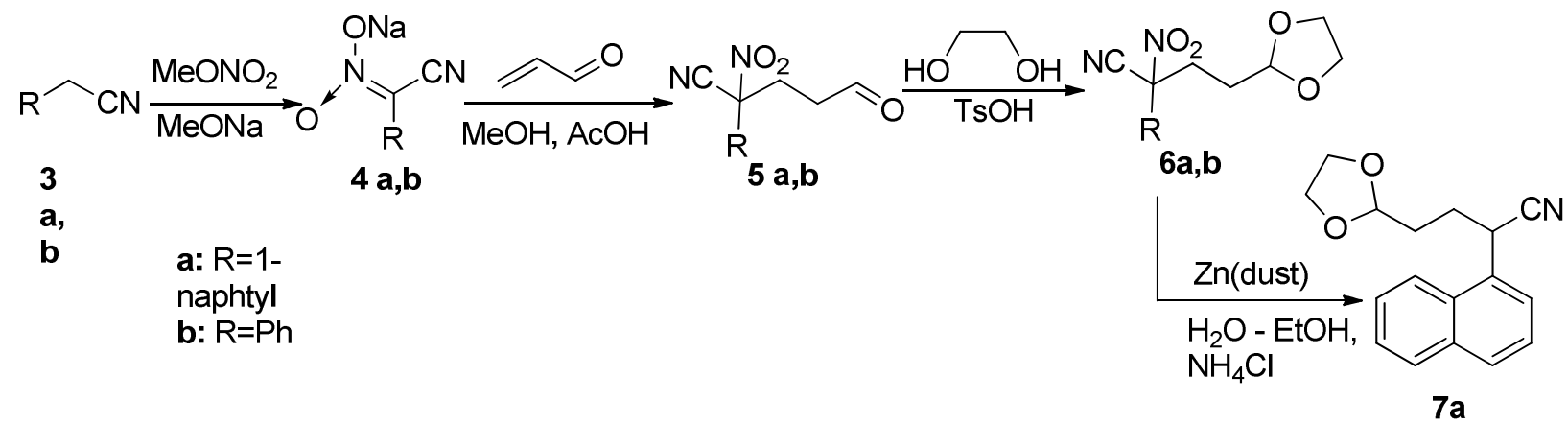

Scheme 3. Synthesis and reduction of 1,4-nitroaldehyde 6.

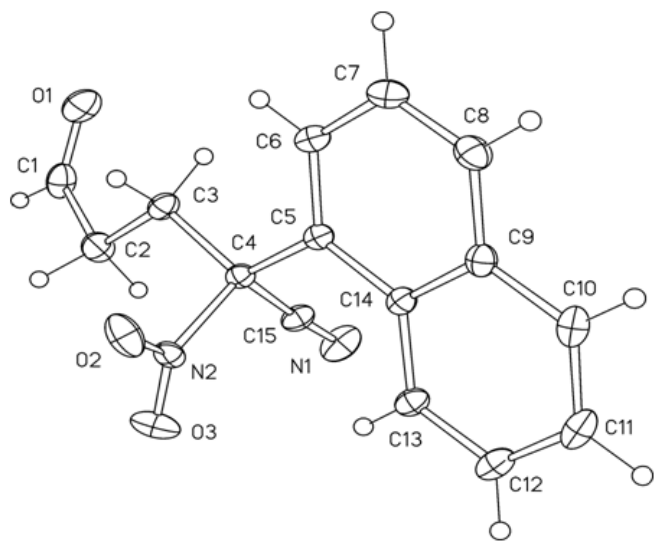

Figure 1. The molecular structure of 5a.

Further reaction of $\mathbf{5 a}, \mathbf{b}$ with ethylene glycol in the presence of catalytic amount of $\mathrm{TsOH}$ gives 1,3-dioxolanes 6a,b. Reduction of nitro group in 6a by zinc dust in water-ethanol solution in the presence of $\mathrm{NH}_{4} \mathrm{Cl}$ unexpectedly leads to 4-(1,3-dioxolan-2-yl)-2-(naphthalen-1yl)butanenitrile $7 \mathbf{a}$ instead of the desired hydroxylamine. Probably, the reason of this transformation is the presence of electron withdrawing cyano group, that stabilize anion product of elimination reaction of initially formed anion-radical - product of one-electron 
reduction. Hereby, in this case elimination of anion-radical is faster than its protonation (Scheme 4).
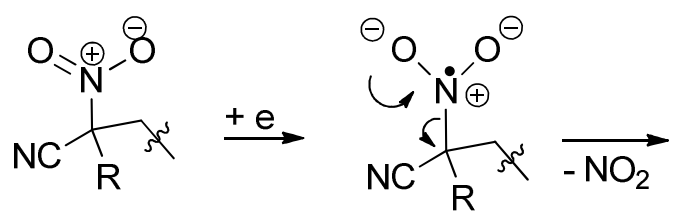<smiles>[R]C([CH][CH])C[CH]</smiles>

Scheme 4. Supposed mechanism of 7a formation.

Thus, we failed to insert the fluorescent fragment into a molecule that may be a precursor of desired heterocycle and the next step of our work was the attempts of introducing of fluorescent moiety using reactivity of functional groups already presented in suitable heterocycle.

We succeed to insert fluorescent moiety into the aldonitrone molecule with the use of 2(ethoxycarbonyl)-2-methyl-3,4-dihydro-2H-pyrrole 1-oxide (EMPO) ${ }^{1} \mathbf{8}$ as starting material. Commercially available compound $\mathbf{8}$ nowadays is used as a spin trap that readily reacts with superoxide radical. ${ }^{12}$ We found that reaction of $\mathbf{8}$ with excess of ethylenediamine (Scheme 5) gives aminoamide 9 and further reaction of 9 with 2,4-dinitrofluorobenzene or 5(dimethylamino)naphthalene-1-sulfonyl chloride (dansyl chloride) in the presence of triethylamine yields $\mathbf{1 0 a}$ and $\mathbf{1 0 b}$ respectively.

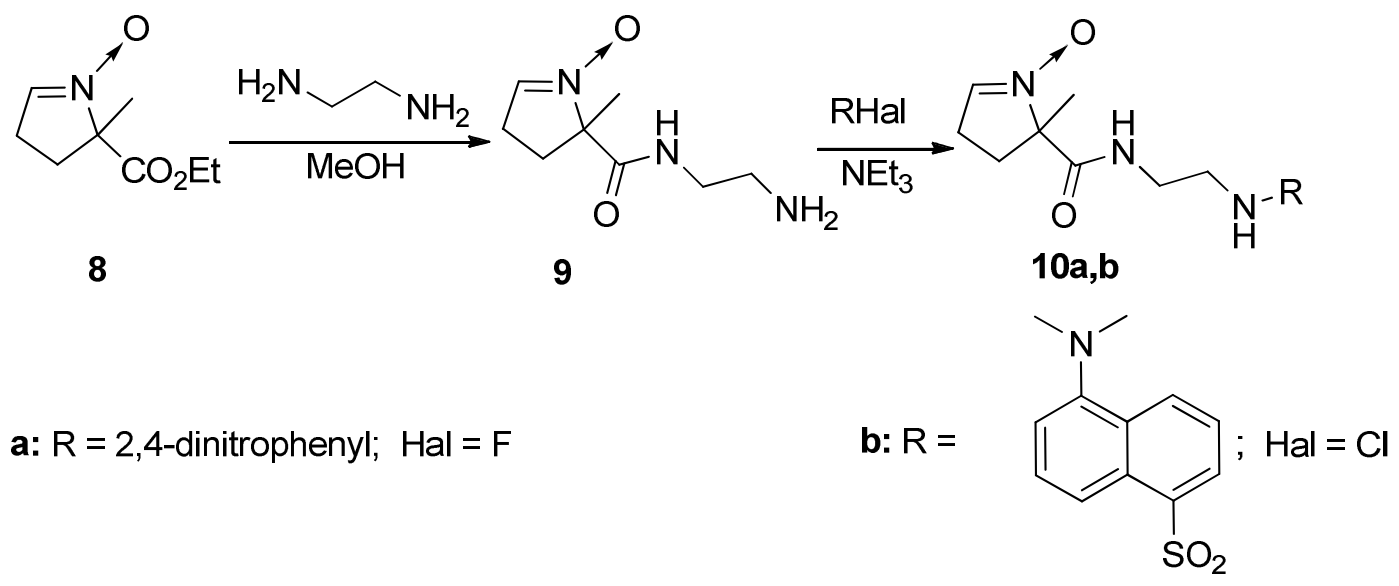

Scheme 5. Synthesis of 10a-b from EMPO.

Nitrone $\mathbf{8}$ could be very promising precursor for fluorescent spin traps, since variation of fluorescent moiety on the last step of synthesis allows to produce spin traps with different physical and chemical properties. Limitations of this approach are high commercial value of $\mathbf{8}$ and its limited availability.

Another way of introduction of the fluorescent moiety is the synthetic sequence including reaction of nitrones with appropriate organometallic compounds followed by oxidation of 
hydroxylamines formed into nitrones. This method was previously widely used in the synthesis of pyrrolidine (proxyl) nitroxides. ${ }^{13,14}$

Reaction of benzylmagnesium chloride as a model reagent with nitrone $\mathbf{1 1}$ leads to hydroxylamine 12a, which was oxidized without purification by manganese dioxide into nitrone 13a (Scheme 6). Similarly, nitrone 13b was obtained by reaction of (naphthalen-1ylmethyl)magnesium chloride with nitrone 11. Addition of naphthalen-1-ylmagnesium bromide to nitrone $\mathbf{1 1}$ did not proceed probably due to steric hindrance. In this case cross-metallation of methyl group in the position 5 of heterocycle seems to proceed with further self-condensation products formation. ${ }^{15}$

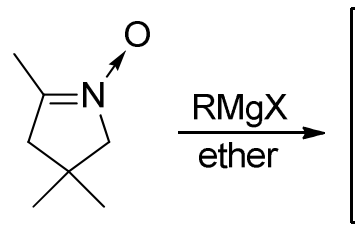

11<smiles>[R]C1(C)CC(C)(C)CN1O</smiles>

$12 a, b, c$<smiles></smiles>

13a,b a: $\mathrm{R}=\mathrm{PhCH}_{2}$

b: $\mathrm{R}=$ (naphthalen-1-yl)- $\mathrm{CH}_{2}$

c: $R=$ naphthalen-1-yl

Scheme 6. Synthesis of nitrones 13a-b.

The last approach examined was based on the condensation of nitrone 11 with aldehydes ${ }^{16}$ containing fluorescent moiety and further transformations of formed $\alpha$, $\beta$-unsaturated nitrones (Scheme 7).
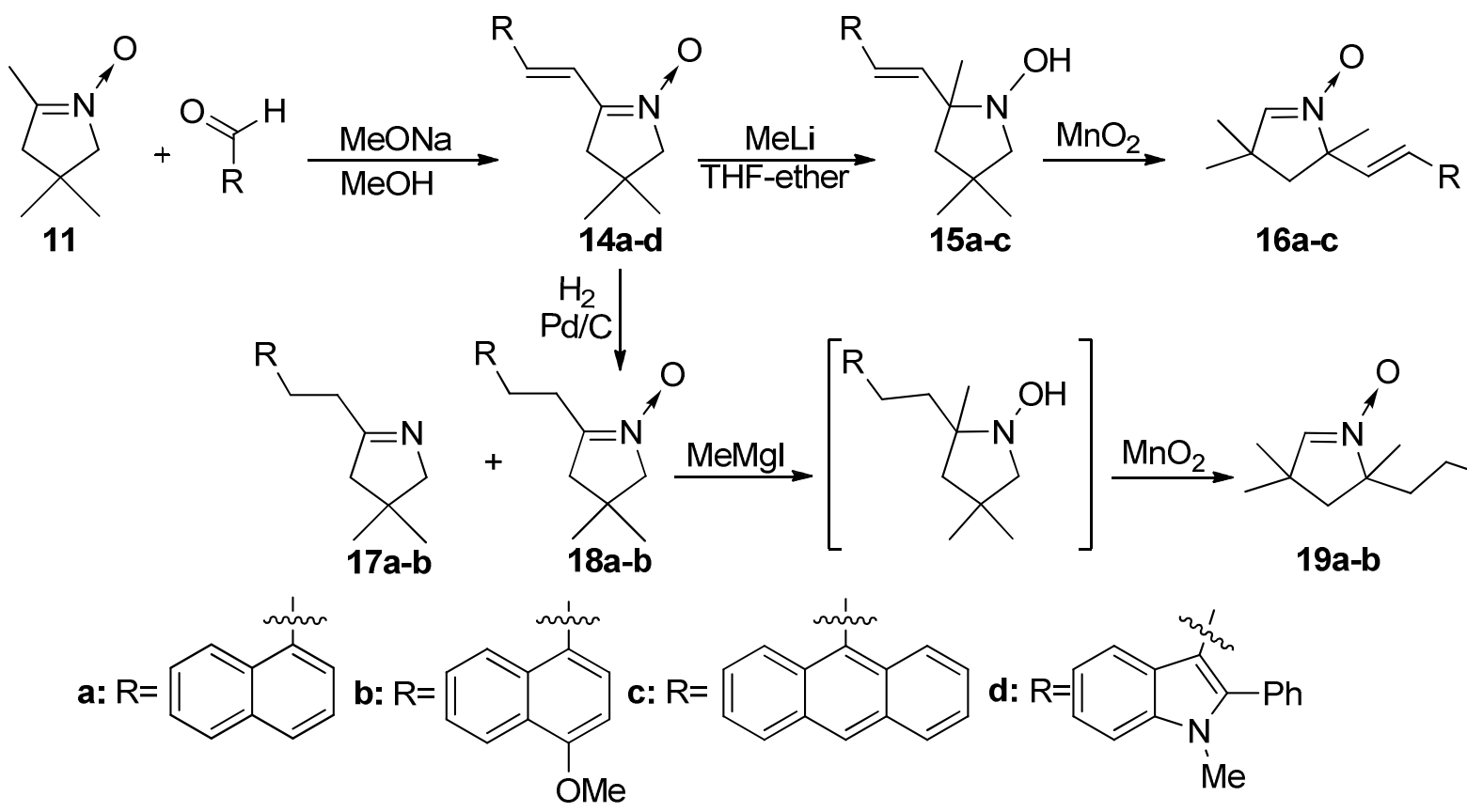

d:<smiles>[R]c1ccc2c(C(C)(C)C)c(-c3ccccc3)n(C)c2c1</smiles>

Scheme 7. Synthesis of nitrones 16a-c and 19a-b. 
It was shown that nitrone $\mathbf{1 1}$ easily reacts with series of aromatic aldehydes with formation of 14. It should be noted, that the resulting products possessed trans-configuration of double bond. Molecular structures of 14a and 14d are shown on the Figures 2 and 3 respectively. Further modification of nitrones $\mathbf{1 4}$ was carried out by two different ways.

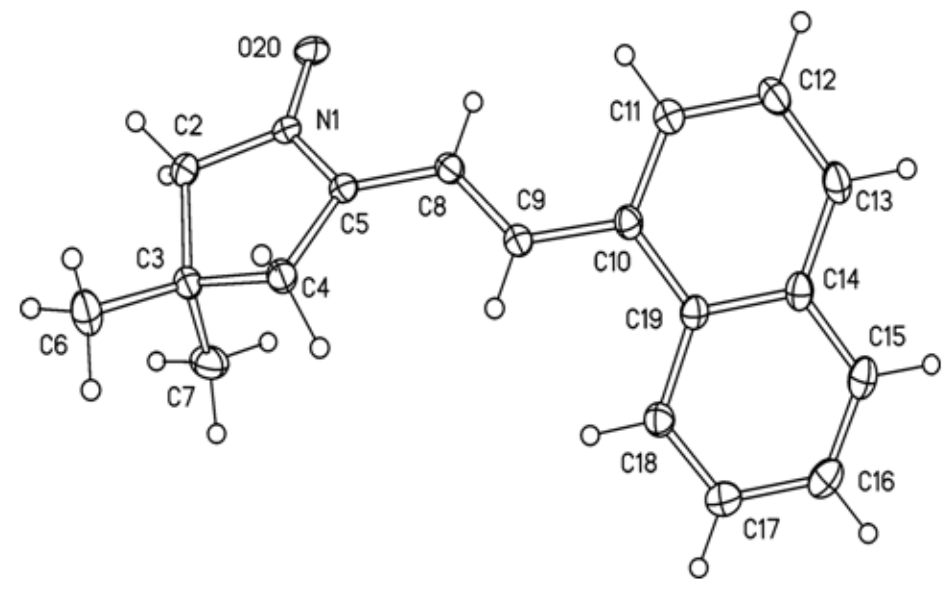

Figure 2. The molecular structure of $\mathbf{1 4 a}$.

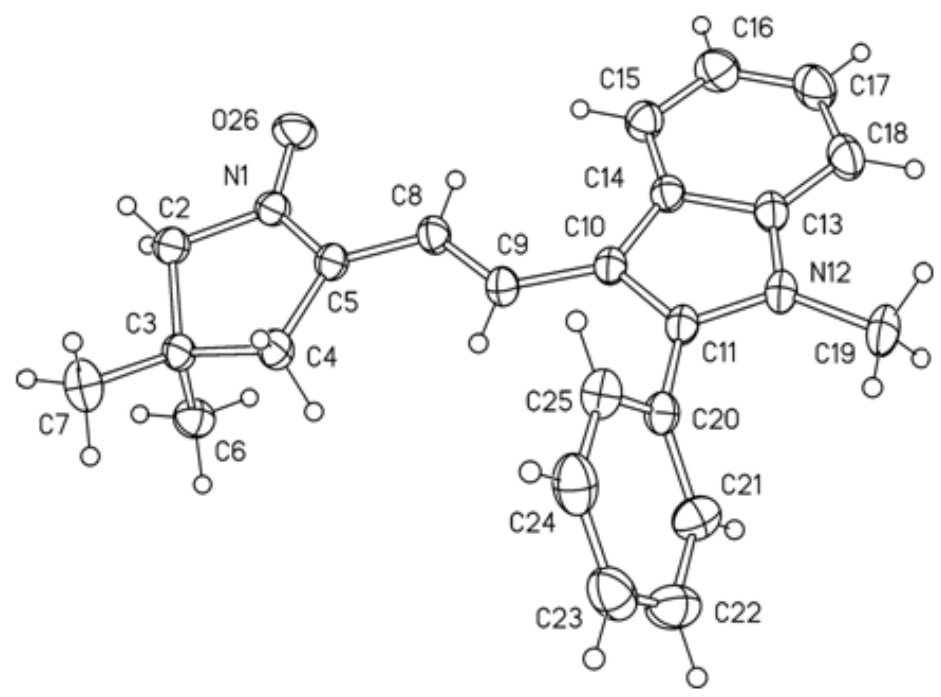

Figure 3. The molecular structure of $\mathbf{1 4 d}$.

When 14a-c reacted with methyllithium in the THF - ether solution hydroxylamines 15a-c were formed. They were oxidized without isolation in individual form into nitrones 16a-c.

When 14a-b were hydrogenated at atmospheric pressure in the presence of $\mathrm{Pd} / \mathrm{C}$ catalyst, nitrones 18a-b were formed along with pyrrolines $\mathbf{1 7 a - b}$ as a by-products. Further treatment of 
18a-b by methylmagnesium iodide followed by oxidation of formed hydroxylamines leads to aldonitrones 19a-b.

Thus, nitrone $\mathbf{1 1}$ was shown to be a convenient precursor for the series fluorescent nitrones 16 and 19 possessed different absorption and emission maxima. An applicability of synthesized fluorescent nitrones as spin traps will be studied at an early day.

\section{Experimental Section}

General. All spectra and elemental analyses were carried out by the Chemical Service Center of the Novosibirsk Institute of Organic Chemistry. ${ }^{1} \mathrm{H}$ and ${ }^{13} \mathrm{C}$ NMR spectra were recorded on Bruker AV-300 and AV-400 spectrometers; solvents were used as internal standards. IR spectra were recorded on Bruker IFS 66 spectrometer for $\mathrm{KBr}$ pellets (concentration $0.25 \%$, pellet thickness $1 \mathrm{~mm}$ ). Mass spectra were recorded on DFS (Thermo Electron) spectrometer and on Bruker micrOTOF-Q spectrometer in a direct input. GC-MS spectra were recorded on HP1800A (Hewlett-Packard) spectrometr. Fluorescence spectra were recorded on Cary Eclipse fluorimeter for $10^{-5} \mathrm{M}$ solutions in chloroform. Melting points were measured in a sealed capillary. Silufol UV 254 and Merck Kieselgel 60 F254 plates were used for TLC monitoring. Chromatography was carried out using "Merck" silica gel $(0.063-0.100 \mathrm{~mm})$ for column chromatography.

The X-ray diffraction experiments for compounds 5a, 14a, 14d were carried out on Bruker KAPPA APEX II diffractometer (graphite-monochromated Mo K $\alpha$ radiation). Reflection intensities were corrected for absorption by SADABS program. The structures was solved by direct methods using the SHELXS-97 program ${ }^{17}$ and refined by anisotropic (isotropic for all $\mathrm{H}$ atoms) full-matrix least-squares method against $F^{2}$ of all reflections using SHELX-97 program. The positions of the hydrogen were calculated geometrically and refined in riding model.

The obtained crystal structure was analyzed for short contacts between non-bonded atoms and hydrogen bonding (table 1) using the PLATON program. ${ }^{18}$ Crystallographic data for the structures of compounds 5a, 14a, 14d in this paper have been deposited at the Cambridge Crystallographic Data Centre as supplementary publication no. CCDC 917551 - 917553. Copy of the data can be obtained, free of charge, on application to CCDC, 12 Union Road, Cambridge CB21EZ, UK (fax: +44 1223336033 or e-mail: deposit@ccdc.cam.ac.uk; internet: www.ccdc.cam.ac.uk).

Reaction of ethyl 2-bromo-2-(naphthalene-1-yl)acetate with sodium nitrite. To a stirred solution of sodium nitrtite $(0.55 \mathrm{~g}, 7.99 \mathrm{mmol})$ and phloroglucinol $(0.6 \mathrm{~g}, 4.74 \mathrm{mmol})$ in anhydrous DMF $(7.5 \mathrm{~mL})$ a solution of ethyl 2-bromo-2-(naphthalene-1-yl)acetate $(1.3 \mathrm{~g}, 4.44$ mmol) in anhydrous DMF ( $2 \mathrm{~mL})$ was added. Dark solution was kept for 17 hours at room temperature and poured into $20 \mathrm{~mL}$ of ice-cold water. Resulted solution was extracted by ether $(3 \times 20 \mathrm{~mL})$ and the combined extract was washed by $30 \mathrm{~mL}$ of saturated sodium hydrocarbonate solution, dried with $\mathrm{MgSO}_{4}$ and concentrated in vacuo. According to GC-MS and ${ }^{1} \mathrm{H}$ NMR 
analysis formation of ethyl 2-nitro-2-(naphthalene-1-yl)acetate didn't proceed. The main product of reaction was ethyl 2-hydroxy-2-(naphthalene-1-yl)acetate. In the ${ }^{1} \mathrm{H} \mathrm{NMR}(300.13 \mathrm{MHz}$, $\mathrm{CDCl}_{3}$ ) spectrum of the reaction mixture the following characteristic signals was observed: $\delta$ 1.12 (t, $J_{H, H}^{3} 7.2 \mathrm{~Hz}, 3 \mathrm{H}, \mathrm{CH}_{3} \mathrm{CH}_{2}$ ), 3.67 (br.s, $\left.1 \mathrm{H}, \mathrm{OH}\right), 4.10-4.30$ (m, 2H, $\mathrm{CH}_{3} \mathrm{CH}_{2}$ ), 5.79 (s, $1 \mathrm{H}, \mathrm{CHOH}), 7.40-8.10$ (m, 7H, arom). This signals correspond to spectrum of 2-hydroxy-2(naphthalene-1-yl)acetate. ${ }^{19}$

Sodium cyano(naphthalen-1-yl)methyleneazinate (4a) was synthesized as described previously. ${ }^{20} \operatorname{IR}\left(v_{\max }, \mathrm{cm}^{-1}\right): 2203(\mathrm{C} \equiv \mathrm{N}), 1475\left(\mathrm{NO}_{2} \mathrm{Na}\right), 1454,1332,1266,1250,1094,947$, 769. ${ }^{1} \mathrm{H}$ NMR $\left(400.13 \mathrm{MHz},\left(\mathrm{CD}_{3}\right)_{2} \mathrm{SO}\right): \delta 7.47-7.51(\mathrm{~m}, 4 \mathrm{H}), 7.80-7.84(\mathrm{~m}, 2 \mathrm{H}), 7.89-7.92$ (m, 1H, arom.). ${ }^{13} \mathrm{C}$ NMR (100.61 MHz, $\left.\left(\mathrm{CD}_{3}\right)_{2} \mathrm{SO}\right): \delta 121.6(\underline{\mathrm{CN}}), 125.4,125.56,125.59,126.0$, $127.4,127.8,128.1,130.5,131.0,133.4$ (arom.), 166.9 (br. s., $\underline{C}=\mathrm{NO}_{2} \mathrm{Na}$ ).

Sodium cyano(phenyl)methyleneazinate (4b) was obtained by the procedure described for $\mathbf{4 a}$ from $3 \mathbf{b}(2.46 \mathrm{~g}, 21 \mathrm{mmol})$. Colorless crystals, yield $58 \%, 2.2 \mathrm{~g} ; \mathrm{mp}$ above $250{ }^{\circ} \mathrm{C} ; \mathrm{IR}\left(v_{\max }, \mathrm{cm}^{-1}\right)$ : 2212, 1503, $1477\left(\mathrm{NO}_{2} \mathrm{Na}\right), 1436,1353,1232,751 .{ }^{1} \mathrm{H} \mathrm{NMR}\left(300.13 \mathrm{MHz},\left(\mathrm{CD}_{3}\right)_{2} \mathrm{SO}\right): \delta 6.96-$ $7.03(\mathrm{~m}, 1 \mathrm{H}), 7.22-7.30(\mathrm{~m}, 1 \mathrm{H}), 7.77-7.82(\mathrm{~m}, 2 \mathrm{H}, \mathrm{Ph}) .{ }^{13} \mathrm{C}$ NMR $\left(75.46,\left(\mathrm{CD}_{3}\right)_{2} \mathrm{SO}\right): \delta 119.9$ $(\underline{\mathrm{CN}}), 122.9,123.4,127.9,133.4(\mathrm{Ph}), 165.8$ (br. s., $\underline{\mathrm{C}}=\mathrm{NO}_{2} \mathrm{Na}$ ).

2-(Naphthalen-1-yl)-2-nitro-5-oxopentanenitrile (5a). Glacial acetic acid $(0.01 \mathrm{~mL}, 1.71$ $\mathrm{mmol})$ and acrolein $(0.057 \mathrm{~mL}, 0.855 \mathrm{mmol})$ were added to a stirred solution of $\mathbf{4 a}(0.20 \mathrm{~g}, 0.855$ $\mathrm{mmol})$ in anhydrous methanol $(0.85 \mathrm{~mL})$. Resulted solution was kept for 24 hours at $45^{\circ} \mathrm{C}$. The solvent was removed on reduced pressure, the residue was diluted with water $(2 \mathrm{~mL})$ and extracted with chloroform $(3 \times 4 \mathrm{~mL})$. Combined extract was dried with $\mathrm{MgSO}_{4}$, the solvent was removed under reduced pressure. The residue was purified by chromatography with a mixture of $\mathrm{CHCl}_{3}-\mathrm{MeOH}(50: 1)$ as eluent. Colorless crystals, yield $150 \mathrm{mg}(65 \%)$, mp $106-108{ }^{\circ} \mathrm{C}$ (from mixture hexane - ethylacetate 3:1, with decomposition); $\operatorname{IR}\left(v_{\max }, \mathrm{cm}^{-1}\right): 2833,2728,1727(\mathrm{C}=\mathrm{O})$, 1576, $1349\left(\mathrm{NO}_{2}\right), 802,776 .{ }^{1} \mathrm{H}$ NMR (400.13 MHz, $\left.\mathrm{CDCl}_{3}\right): \delta 2.79-2.89\left(\mathrm{~m}, 1 \mathrm{H}, \mathrm{CH}_{2} \mathrm{CHO}\right)$, $3.04-3.13$ (m, 1H, $\underline{\mathrm{H}}_{2} \mathrm{CHO}$ ), 3.22 - 3.27 (m, 1H, $\left.\mathrm{C}_{2} \mathrm{CH}_{2} \mathrm{CHO}\right), 7.54-7.67$ (m, 3H), 7.82 $7.85(\mathrm{~m}, 1 \mathrm{H}), 7.93-7.97(\mathrm{~m}, 1 \mathrm{H}), 8.02-8.07$ (m, 2H, arom.), 9.85 (s, 1H, C부). ${ }^{13} \mathrm{C}$ NMR (100.61MHz, $\left.\mathrm{CDCl}_{3}\right): \delta 30.3\left(\underline{\mathrm{CH}}_{2} \mathrm{CHO}\right), 39.4\left(\underline{\mathrm{CH}}_{2} \mathrm{CH}_{2} \mathrm{CHO}\right), 89.1\left(\underline{\mathrm{C}}(\mathrm{CN})\left(\mathrm{NO}_{2}\right)\right), 114.7(\underline{\mathrm{CN}})$, 122.1, 124.9, 126.4, 126.8, 127.1, 128.7, 129.8, 130.0, 134.4 (arom.), 197.5 (ㄷHO). Calcd. for $\mathrm{C}_{15} \mathrm{H}_{12} \mathrm{~N}_{2} \mathrm{O}_{3}$ (268.08): C 67.16, H 4.51, N 10.44. Found: C 67.00, H 4.32, N 10.47.

Crystallographic data for comp 5a: $\mathrm{C}_{15} \mathrm{H}_{12} \mathrm{~N}_{2} \mathrm{O}_{3}, M=268.27$, monoclinic, $P 2{ }_{1} / c, a=11.2063(8)$, $b=10.9032(7), c=10.5235(7) \AA, \beta=91.050(2)^{\circ}, V=1285.6(2) \AA^{3}, Z=4, D_{\text {calcd }}=1.386 \mathrm{~g} \cdot \mathrm{cm}^{-3}$, $\mu(\mathrm{Mo}-\mathrm{K \alpha})=0.099 \mathrm{~mm}^{-1}, \mathrm{~F}(000)=560,\left(\theta 1.82-29.17^{\circ}\right.$, completeness $\left.99.8 \%\right), \mathrm{T} 150(2) \mathrm{K}$, colorless prism, $(0.56 \times 0.16 \times 0.15) \mathrm{mm}^{3}$, transmission $0.9615-0.9895,25142$ measured reflections in index range $-15<=\mathrm{h}<=15,-14<=\mathrm{k}<=14,-14<=1<=13,3472$ independent $\left(R_{\text {int }}=\right.$ 0.0218 ), 203 parameters, 3055 observed $[I>2 \sigma(I)], R_{1}=0.0410, w R_{2}=0.1274$ (all data), GOOF 1.069 , largest diff. peak and hole 0.321 and -0.209 e. $\mathrm{A}^{-3}$. The atom positions of cyano and nitro groups are disordered due to enatiomers placement in the same position with ratio 85:15

2-Nitro-5-oxo-2-phenylpentanenitrile (5b) was synthesized according to the procedure described for $\mathbf{5 a}$ from $\mathbf{4 b}(0.16 \mathrm{~g}, 0.855 \mathrm{mmol})$. Pale yellow oil, yield $66 \%$; IR( $\left(v_{\max }, \mathrm{cm}^{-1}\right): 1726$ 
$(\mathrm{C}=\mathrm{O}), 1570,1452\left(\mathrm{NO}_{2}\right), 1338,640 .{ }^{1} \mathrm{H}$ NMR $\left(400.13 \mathrm{MHz}, \mathrm{CDCl}_{3}\right): \delta 2.69-2.73(\mathrm{~m}, 2 \mathrm{H}$, $\mathrm{C}_{2} \mathrm{CHO}$ ), $2.82-2.90$ (m, 1H, $\underline{\mathrm{C}}_{2} \mathrm{CH}_{2} \mathrm{CHO}$ ), $3.06-3.14$ (m, 1H, $\underline{\mathrm{C}}_{2} \mathrm{CH}_{2} \mathrm{CHO}$ ), $7.44-7.51$ (m, 3H), $7.65-7.68$ (m, 2H, Ph), 9.73 (br. s, $1 \mathrm{H}, \mathrm{CHO}) .{ }^{13} \mathrm{C} \mathrm{NMR}\left(100.61 \mathrm{MHz}, \mathrm{CDCl}_{3}\right): \delta 30.5$ $\left(\underline{\mathrm{CH}_{2}} \mathrm{CH}_{2} \mathrm{CHO}\right), 39.0\left(\underline{\mathrm{CH}}_{2} \mathrm{CHO}\right), 91.3\left(\underline{\mathrm{C}}(\mathrm{CN}) \mathrm{NO}_{2}\right), 114.1(\underline{\mathrm{CN}}), 126.4,129.8,130.7,131.8$ $(\mathrm{Ph}), 197.6$ (ㄷO Calcd. for $\mathrm{C}_{11} \mathrm{H}_{10} \mathrm{~N}_{2} \mathrm{O}_{3}$ (218.07): $\mathrm{C} 60.55, \mathrm{H}$ 4.62, N 12.84. Found: C 60.99, $\mathrm{H}$ 4.83, N 12.63 .

4-(1,3-Dioxolan-2-yl)-2-(naphthalen-1-yl)-2-nitrobutanenitrile (6a). A mixture of 5a (1.0 g, $3.63 \mathrm{mmol})$, ethylene glycol $(0.24 \mathrm{~mL}, 4.37 \mathrm{mmol})$, TsOH $(0.006 \mathrm{~g}, 0.034 \mathrm{mmol})$ and benzene $(1.2 \mathrm{~mL})$ was refluxed with Dean-Stark apparatus until the end of water separation (about 3.5 hours). Then the reaction mixture was diluted by benzene $(5 \mathrm{~mL})$ and washed consequentially by sodium hydrocarbonate saturated solution $(2 \times 5 \mathrm{~mL})$ and water $(2 \times 5 \mathrm{~mL})$. Combined organic extract was dried with $\mathrm{MgSO}_{4}$, and the solvent was removed under reduced pressure. The residue was purified by chromatography with a mixture of $\mathrm{CHCl}_{3}-\mathrm{MeOH}(30: 1)$ as eluent. Colorless crystals, yield $0.71 \mathrm{~g}\left(61 \%\right.$ ); $\mathrm{mp} 111-113{ }^{\circ} \mathrm{C}$ (from mixture hexane - ethylacetate $4: 1$, with decomposition); $\operatorname{IR}\left(v_{\max }, \mathrm{cm}^{-1}\right): 2961,2888,1571\left(\mathrm{NO}_{2}\right), 1147,1027,810,781 .{ }^{1} \mathrm{H}$ NMR (300.13 $\left.\mathrm{MHz}, \mathrm{CDCl}_{3}\right): \delta 1.92-2.04\left(\mathrm{~m}, 1 \mathrm{H}, \mathrm{C}_{2} \mathrm{CH}(\mathrm{O}) \mathrm{O}\right), 2.16-2.28\left(\mathrm{~m}, 1 \mathrm{H}, \mathrm{C}_{2} \mathrm{CH}(\mathrm{O}) \mathrm{O}\right), 3.00-$ $3.07\left(\mathrm{~m}, 2 \mathrm{H}, \mathrm{C}_{2} \mathrm{CH}_{2} \mathrm{CH}(\mathrm{O}) \mathrm{O}\right), 3.89-4.02\left(\mathrm{~m}, 4 \mathrm{H}, \mathrm{OC}_{2} \underline{\mathrm{C}}_{2} \mathrm{O}\right), 5.05\left(\mathrm{t}, 1 \mathrm{H}, J_{H, H}^{3} 4.0 \mathrm{~Hz}\right.$, OC타), $7.50-7.66(\mathrm{~m}, 3 \mathrm{H}), 7.82-7.85(\mathrm{~m}, 1 \mathrm{H}), 7.91-7.95(\mathrm{~m}, 1 \mathrm{H}), 7.99-8.03(\mathrm{~m}, 1 \mathrm{H})$, $8.08-8.13$ (m, $1 \mathrm{H}$, arom.). ${ }^{13} \mathrm{C}$ NMR $\left(100.61 \mathrm{MHz}, \mathrm{CDCl}_{3}\right): \delta 29.1\left(\mathrm{CH}_{2} \mathrm{CH}(\mathrm{O}) \mathrm{O}\right), 31.6$ $\left(\underline{\mathrm{C}} \mathrm{H}_{2} \mathrm{CH}_{2} \mathrm{CH}(\mathrm{O}) \mathrm{O}\right), 65.11 \quad\left(\mathrm{O}_{\mathrm{CH}} \mathrm{CH}_{2} \mathrm{O}\right), 65.14 \quad\left(\mathrm{O}_{2} \mathrm{H}_{2} \mathrm{CH}_{2} \mathrm{O}\right), 89.4 \quad\left(\underline{\mathrm{C}}(\mathrm{CN})\left(\mathrm{NO}_{2}\right)\right), 102.2$ (OㄷHO), 114.7 ( $\underline{\mathrm{CN}}), 122.0,124.6,126.5,126.66,126.72,128.2,129.7,129.8,132.8,134.2$ (arom.). Calcd. for $\mathrm{C}_{17} \mathrm{H}_{16} \mathrm{~N}_{2} \mathrm{O}_{4}$ (312.32): C 65.38, H 5.16, N 8.97. Found: C 65.62, H 5.08, N 9.07 .

4-(1,3-Dioxolan-2-yl)-2-nitro-2-phenylbutanenitrile (6b) was synthesized according to the procedure described for $\mathbf{6 a}$ from $5 \mathbf{b}(0.81 \mathrm{~g}, 3.73 \mathrm{mmol})$. Pale yellow oil, yield $0.69 \mathrm{~g}(72 \%)$; $\operatorname{IR}\left(v_{\max }, \mathrm{cm}^{-1}\right): 2959,2890,1570,1338\left(\mathrm{NO}_{2}\right), 1138,1028,693 .{ }^{1} \mathrm{H} \mathrm{NMR}\left(300.13 \mathrm{MHz}, \mathrm{CDCl}_{3}\right)$ : $\delta$ 1.80-1.90 (m, $\left.2 \mathrm{H}, \mathrm{CH}_{2} \mathrm{CH}(\mathrm{O}) \mathrm{O}\right), 2.61-2.72\left(\mathrm{~m}, 1 \mathrm{H}, \mathrm{CH}_{2} \mathrm{CH}_{2} \mathrm{CHOO}\right), 2.85-2.97(\mathrm{~m}, 1 \mathrm{H}$, $\left.\mathrm{C}_{2} \mathrm{CH}_{2} \mathrm{CH}(\mathrm{O}) \mathrm{O}\right), 3.82-3.95\left(\mathrm{~m}, 4 \mathrm{H}, \mathrm{OC}_{2} \underline{\mathrm{CH}}_{2} \mathrm{O}\right), 4.94$ (t, $\left.1 \mathrm{H}, J_{H, H}^{3} 4.0 \mathrm{~Hz}, \mathrm{OC} \underline{\mathrm{HO}}\right), 7.41-7.49$ (m, 3H), 7.66-7.70 (m, 2H, Ph). ${ }^{13} \mathrm{C}$ NMR $\left(75.46 \mathrm{MHz}, \mathrm{CDCl}_{3}\right): \delta 29.1\left(\mathrm{CH}_{2} \mathrm{CH}_{2} \mathrm{CHO}\right), 32.0$ $\left(\underline{\mathrm{C}} \mathrm{H}_{2} \mathrm{CHO}\right), 65.16\left(\mathrm{O}_{\underline{C}} \mathrm{H}_{2} \mathrm{CH}_{2} \mathrm{O}\right), 65.19\left(\mathrm{OCH}_{2} \underline{\mathrm{CH}}_{2} \mathrm{O}\right), 92.0\left(\underline{\mathrm{C}}(\mathrm{CN}) \mathrm{NO}_{2}\right), 102.2(\mathrm{O} \underline{\mathrm{CHO}}), 114.3$ $(\underline{\mathrm{CN}}), 126.5,129.6,131.1,131.5(\mathrm{Ph})$. Calcd. for $\mathrm{C}_{13} \mathrm{H}_{14} \mathrm{~N}_{2} \mathrm{NaO}_{4}: \mathrm{m} / z 285.0850$ [M+Na] $]^{+}$. Found: $\mathrm{m} / \mathrm{z} 285.0846[\mathrm{M}+\mathrm{Na}]^{+}$.

4-(1,3-Dioxolan-2-yl)-2-(naphthalen-1-yl)butanenitrile (7a). To a cooled stirred mixture of 6a $(0.574 \mathrm{~g}, 1.84 \mathrm{mmol}), \mathrm{NH}_{4} \mathrm{Cl}(0.108 \mathrm{~g}, 2.02 \mathrm{mmol}), \mathrm{EtOH}(6 \mathrm{~mL})$ and water $(6 \mathrm{~mL})$ zinc dust $(0.499 \mathrm{~g}, 7.67 \mathrm{mmol})$ was added by small portion maintaining the temperature at $10^{\circ} \mathrm{C}$. After the addition of zinc, cooling was removed and mixture was vigorously stirred for 2 hours at room temperature. The precipitate was filtered off and washed with methanol $(3 \times 4 \mathrm{~mL})$. The filtrate was concentrated in vacuo and the residue was diluted with water $(5 \mathrm{~mL})$ and extracted by chloroform $(4 \times 5 \mathrm{~mL})$. Combined organic extract was dried with $\mathrm{MgSO}_{4}$ and the solvent was removed under reduced pressure. The residue was purified by chromatography with a mixture of 
$\mathrm{CHCl}_{3}-\mathrm{MeOH}(40: 1)$ as eluent. Pale yellow oil, yield $0.15 \mathrm{~g}(31 \%) .{ }^{1} \mathrm{H}$ NMR $(400.13 \mathrm{MHz}$, $\mathrm{CDCl}_{3}$ ): $\delta$ 1.93-1.96 (m, 2H, $\underline{\mathrm{H}}_{2} \mathrm{CHOO}$ ), 2.09 - 2.21 (m, 2H, $\underline{\mathrm{C}}_{2} \mathrm{CH}$-arom.), 3.81 - 3.95 (m, $\left.4 \mathrm{H}, \mathrm{OC}_{2} \mathrm{C}_{2} \mathrm{O}\right), 4.69$ (dd, $1 \mathrm{H}, J_{H, H}^{3} 5.4 \mathrm{~Hz}, J_{H, H}^{3} 9.3 \mathrm{~Hz}, \mathrm{C} \underline{\mathrm{H}}$-arom.), 4.91 (t, $1 \mathrm{H}, J_{H, H}^{3} 4.3 \mathrm{~Hz}$, OC브), $7.43-7.58(\mathrm{~m}, 3 \mathrm{H}), 7.66-7.69(\mathrm{~m}, 1 \mathrm{H}), 7.79-7.83(\mathrm{~m}, 1 \mathrm{H}), 7.86-7.89(\mathrm{~m}, 1 \mathrm{H}), 7.91$ - $7.95\left(\mathrm{~m}, 1 \mathrm{H}\right.$, arom). ${ }^{13} \mathrm{C} \mathrm{NMR}\left(75.46 \mathrm{MHz}, \mathrm{CDCl}_{3}\right): \delta 28.9\left(\mathrm{CH}_{2} \mathrm{CH}(\mathrm{O}) \mathrm{O}\right), 31.0\left(\underline{\mathrm{CH}}_{2} \mathrm{CH}-\right.$ arom.), 34.1 (ㄷH-arom), $64.95\left(\mathrm{OCH}_{2} \mathrm{CH}_{2} \mathrm{O}\right), 65.02\left(\mathrm{OCH}_{2} \underline{\mathrm{CH}}_{2} \mathrm{O}\right), 103.5(\mathrm{O} \underline{\mathrm{CHO}}), 121.0(\underline{\mathrm{CN}})$, 122.2 , 125.48, 125.52, 126.1, 126.9, 129.0, 129.3, 130.0, 131.6, 134.0 (arom).

2-(2-Aminoethylcarbamoyl)-2-methyl-3,4-dihydro-2H-pyrrole 1-oxide 9. A solution of ethylenediamine $(3.9 \mathrm{~mL}, 58 \mathrm{mmol}$ ) and 2-(ethoxycarbonyl)-2-methyl-3,4-dihydro-2H-pyrrole 1oxide $8(1 \mathrm{~g}, 5.8 \mathrm{mmol})$ in methanol $(2 \mathrm{~mL})$ was kept for 48 hours at room temperature and then concentrated in vacuo. The residue was purified by chromatography with a mixture of $\mathrm{CHCl}_{3}-$ methanol $-\mathrm{NEt}_{3}(30: 10: 1)$ as eluent. Pale yellow oil, yield $42 \%, 0.45 \mathrm{~g} ; \operatorname{IR}\left(v_{\max }, \mathrm{cm}^{-1}\right): 3327(\mathrm{~N}-$ $\mathrm{H}), 2936,1660(\mathrm{C}=\mathrm{O}), 1529,752 .{ }^{1} \mathrm{H}$ NMR $\left(300.13 \mathrm{MHz}, \mathrm{CDCl}_{3}\right): \delta 1.63\left(\mathrm{~s}, 3 \mathrm{H}, \mathrm{CH}_{3}\right), 2.06(\mathrm{dt}$, $2 \mathrm{H}, J_{H, H}^{3} 7.9 \mathrm{~Hz}, J_{H, H}^{2} 13.5 \mathrm{~Hz}, \mathrm{C}_{2} \mathrm{C}\left(\mathrm{CH}_{3}\right) \mathrm{CO}$ ), 2.53 (dt, $2 \mathrm{H}, J_{H, H}^{3} 7.9 \mathrm{~Hz}, J_{H, H}^{3} 2.7 \mathrm{~Hz}$, $\underline{\mathrm{CH}}_{2} \mathrm{CH}=\mathrm{N}$ ), $2.75\left(\mathrm{t}, 2 \mathrm{H}, J_{H, H}^{3} 6.2 \mathrm{~Hz}, \underline{\mathrm{CH}}_{2} \mathrm{CH}_{2} \mathrm{NH}_{2}\right.$ ), $2.87-2.96$ (m, 2H, $\underline{\mathrm{H}}_{2}$ ), 3.23 (q, 2H, $\left.J_{H, H}^{3} 6.2 \mathrm{~Hz}, \mathrm{CH}_{2} \mathrm{CH}_{2} \mathrm{NH}_{2}\right), 6.92\left(\mathrm{t}, 1 \mathrm{H}, \vec{J}_{H, H}^{3} 2.7 \mathrm{~Hz}, \mathrm{C} \underline{\mathrm{H}}=\mathrm{N}\right), 8.56$ (br. s, $\left.1 \mathrm{H}, \underline{\mathrm{H}}\right) .{ }^{13} \mathrm{C} \mathrm{NMR}$ (75.46MHz, $\left.\mathrm{CDCl}_{3}\right): \delta 24.1\left(\mathrm{CH}_{3}\right), 24.6\left(\underline{\mathrm{CH}}_{2} \mathrm{CH}_{2} \mathrm{NH}_{2}\right), 30.4\left(\mathrm{CH}_{2} \underline{\mathrm{CH}}_{2} \mathrm{NH}_{2}\right), 41.3\left(\mathrm{~N}=\mathrm{CHCH}_{2}\right)$, $42.4\left(\underline{\mathrm{CH}_{2} \mathrm{C}}\left(\mathrm{CH}_{3}\right) \mathrm{CO}\right), 78.6\left(\underline{\mathrm{C}}\left(\mathrm{CH}_{3}\right) \mathrm{CO}\right), 137.1(\mathrm{~N}=\underline{\mathrm{CH}}), 170.9(\mathrm{C}=\mathrm{O})$. Calcd. for $\mathrm{C}_{8} \mathrm{H}_{15} \mathrm{~N}_{3} \mathrm{O}_{2}$ : $m / z, 185.1159[\mathrm{M}]^{+}$. Found: $m / z$ 185.1160 [M] ${ }^{+}$.

2-(2-(2,4-Dinitrophenylamino)ethylcarbamoyl)-2-methyl-3,4-dihydro-2H-pyrrole 1-oxide 10a. A solution of 2,4-dinitrofluorobenzene $(0.093 \mathrm{~g}, 0.5 \mathrm{mmol})$ and triethylamine $(0.08 \mathrm{~mL}, 0.5$

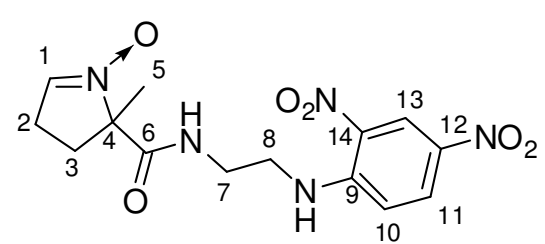
$\mathrm{mmol})$ in anhydrous acetonitrile $(2.5 \mathrm{~mL})$ was added dropwise to a solution of $9(0.1 \mathrm{~g}, 0.5 \mathrm{mmol})$ in anhydrous acetonitrile $(10 \mathrm{~mL})$. The reaction mixture was stirred for 1.5 hours at room temperature and then concentrated in vacuo. The residue was diluted with chloroform $(10 \mathrm{~mL})$ and washed with brine.

Combined organic extract was dried with $\mathrm{MgSO}_{4}$ and the solvent was removed under reduced pressure. Product was purified by TLC on alumina with chloroform as eluent. Bright yellow oil, yield $0.097 \mathrm{~g}(55 \%)$; IR $\left(v_{\max }, \mathrm{cm}^{-1}\right): 3359(\mathrm{~N}-\mathrm{H}), 1676(\mathrm{C}=\mathrm{O}), 1618,1607(\mathrm{C}=\mathrm{N}), 1524\left(\mathrm{NO}_{2}\right)$, $1335\left(\mathrm{NO}_{2}\right) .{ }^{1} \mathrm{H}$ NMR $\left(300.13 \mathrm{MHz}, \mathrm{CDCl}_{3}\right): \delta 1.67\left(\mathrm{~s}, 3 \mathrm{H}, \mathrm{CH}_{3}\right), 2.15\left(\mathrm{~m}, 1 \mathrm{H}, \mathrm{C}(2) \mathrm{H}_{2}\right), 2.60(\mathrm{t}$, $2 \mathrm{H}, J_{H, H}^{3} 6.9 \mathrm{~Hz}, \mathrm{C}(3) \mathrm{H}_{2}, 2.89-2.96\left(\mathrm{~m}, 1 \mathrm{H}, \mathrm{C}(2) \mathrm{H}_{2}\right), 3.13\left(\mathrm{~m}, 4 \mathrm{H}, \mathrm{CH}_{2} \mathrm{CH}_{2} \mathrm{NH}\right), 6.96$ (br. s, $1 \mathrm{H}, \mathrm{C}(1) \underline{\mathrm{H}}), 7.03\left(\mathrm{~d}, 1 \mathrm{H}, J_{H, H}^{3} 9.5 \mathrm{~Hz}, \mathrm{H}(10)\right), 8.25$ (dd, $\left.1 \mathrm{H}, J_{H, H}^{3} 2.5 \mathrm{~Hz}, J_{H, H}^{3} 9.5 \mathrm{~Hz}, \mathrm{H}(11)\right)$, 8.70 (br. c, $1 \mathrm{H}, \mathrm{C}(9)-\mathrm{N} \underline{\mathrm{H}}), 8.95$ (br. s, $1 \mathrm{H}, \mathrm{CON} \underline{\mathrm{H}}$ ), 9.08 (d, $\left.1 \mathrm{H}, J^{3}{ }_{H, H} 2.5 \mathrm{~Hz}, \mathrm{H}(13)\right) .{ }^{13} \mathrm{C} \mathrm{NMR}$ $\left(75.46 \mathrm{MHz}, \mathrm{CDCl}_{3}\right): \delta 24.2(\mathrm{C}(8)), 24.7(\mathrm{C}(7)), 37.9(\mathrm{C}(3)), 43.1(\mathrm{C}(2)), 46.3(\mathrm{C}(5)), 78.5$ (C(4)), 114.1 (C(10)), 124.3 (C(13)), 130.5 (C(11)), 130.8 (C(14)), 136.3 (C(12)), 137.8 (C(9)), $148.5(\mathrm{C}(1)), 172.1(\mathrm{C}(6))$.

\section{2-(2-(5-(Dimethylamino)naphthalene-1-sulfonamido)ethylcarbamoyl)-2-methyl-3,4-}

dihydro-2H-pyrrole 1-oxide 10b was synthesized according to the procedure described for 10a from $9(0.1 \mathrm{~g}, 0.5 \mathrm{mmol})$ and dansyl chloride $(0.135 \mathrm{~g}, 0.5 \mathrm{mmol})$. Pale yellowish-green oil, yield $0.059 \mathrm{~g}(28 \%)$; IR( $\left(v_{\max }, \mathrm{cm}^{-1}\right): 3396(\mathrm{~N}-\mathrm{H}), 2940,2863,1669(\mathrm{C}=\mathrm{O}), 1542(\mathrm{C}=\mathrm{N}), 1453,1320$ 


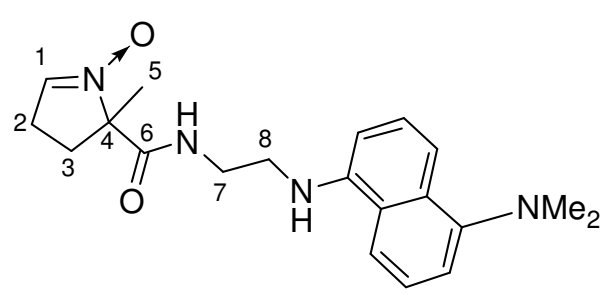

$(\mathrm{S}=\mathrm{O}), 1144(\mathrm{~S}=\mathrm{O}), 792,625,570 .{ }^{1} \mathrm{H}$ NMR $(300.13$ $\left.\mathrm{MHz}, \mathrm{CDCl}_{3}\right): \delta 1.55\left(\mathrm{~s}, 3 \mathrm{H}, \underline{\mathrm{C}}_{3}\right), 2.01\left(\mathrm{dt}, 1 \mathrm{H}, J_{H, H}^{3} 8.0\right.$ $\left.\mathrm{Hz}, J_{H, H}^{2} 13.4 \mathrm{~Hz}, \mathrm{C}(3) \underline{\mathrm{H}}\right), 2.50\left(\mathrm{dt}, 1 \mathrm{H}, J_{H, H}^{3} 7.6 \mathrm{~Hz}, J_{H, H}^{2}\right.$ $\left.2.7 \mathrm{~Hz}, \mathrm{C}(2) \underline{\mathrm{H}}_{2}\right), 2.79-2.90(\mathrm{~m}, 1 \mathrm{H}, \mathrm{C}(3) \underline{\mathrm{H}}), 2.83$ (s, $6 \mathrm{H}$, $\left.\mathrm{N}\left(\mathrm{C}_{3}\right)_{2}\right), 3.00\left(\mathrm{t}, 2 \mathrm{H}, J_{H, H}^{3} 6.0 \mathrm{~Hz}, \mathrm{C}(8) \underline{\mathrm{H}}_{2}\right), 3.30(\mathrm{q}, 2 \mathrm{H}$, $\left.J_{H, H}^{3} 6.0 \mathrm{~Hz}, \mathrm{C}(7) \underline{\mathrm{H}}_{2}\right), 6.69$ (br. s, $1 \mathrm{H}, \mathrm{SO}_{2} \mathrm{NH}$ ), $7.04(\mathrm{t}$, $\left.1 \mathrm{H}, J_{H, H}^{3} 2.7 \mathrm{~Hz}, \mathrm{C}(1) \underline{\mathrm{H}}\right), 7.11\left(\mathrm{~d}, 1 \mathrm{H}, J_{H, H}^{3} 7.5 \mathrm{~Hz}\right), 7.42-7.51(\mathrm{~m}, 2 \mathrm{H}), 8.16\left(\mathrm{dd}, 1 \mathrm{H}, J_{H, H}^{3} 7.3\right.$ $\left.\mathrm{Hz}, J_{H, H}^{4} 1.2 \mathrm{~Hz}\right), 8.27\left(\mathrm{dt}, 1 \mathrm{H}, J_{H, H}^{3} 8.7 \mathrm{~Hz}, J_{H, H}^{4} 0.9 \mathrm{~Hz}\right), 8.47\left(\mathrm{dt}, 1 \mathrm{H}, J_{H, H}^{3} 8.5 \mathrm{~Hz}, J_{H, H}^{4} 0.9\right.$ $\mathrm{Hz}$, arom.), 8.54 (br. t, $\left.1 \mathrm{H}, J_{H, H}^{3} 5.8 \mathrm{~Hz}, \mathrm{CON} \underline{\mathrm{H}}\right) .{ }^{13} \mathrm{C} \mathrm{NMR}\left(75.46 \mathrm{MHz}, \mathrm{CDCl}_{3}\right): \delta 23.6(\mathrm{C}(6))$, $24.9(\mathrm{C}(2)), 30.5(\mathrm{C}(7)), 39.2(\mathrm{C}(3)), 43.1(\mathrm{C}(8)), 45.5\left(\mathrm{~N}\left(\mathrm{CH}_{3}\right)_{2}\right), 78.7(\mathrm{C}(4)), 115.2,119.1$, 123.3, 128.3, 129.4, 129.7, 130.0, 130.3, 135.1 (arom.), 138.8 (C(1)), 152.0 (arom.), 171.3 (C(5)). Calcd. for $\mathrm{C}_{20} \mathrm{H}_{26} \mathrm{~N}_{4} \mathrm{SO}_{4}: \mathrm{m} / z 418.1669[\mathrm{M}]^{+}$. Found: $\mathrm{m} / z$ 418.1664 [M] $]^{+}$. Absorption maximum $-342 \mathrm{~nm}$, emission maximum $-496 \mathrm{~nm}$.

2-Benzyl-2,4,4-trimethyl-3,4-dihydro-2H-pyrrole 1-oxide 13a. To a solution of benzylmagnesium chloride, prepared from magnesium $(0.236 \mathrm{~g}, 9.84 \mathrm{mmol})$ and benzyl chloride $(1.43 \mathrm{~g}, 9.50 \mathrm{mmol})$ in anhydrous ether $(11 \mathrm{~mL})$, a solution of 3,3,5-trimethyl-3,4-dihydro-2Hpyrrole 1-oxide $11(1.0 \mathrm{~g}, 7.87 \mathrm{mmol})$ in anhydrous ether $(1.5 \mathrm{~mL})$ was added. The reaction mixture was stirred for 0.7 hours at room temperature and the excess of Grignard reagent was decomposed by addition of saturated ammonium chloride solution $(10 \mathrm{~mL})$. Ether solution was separated and water layer was extracted with ether $(5 \times 20 \mathrm{~mL})$. Combined organic phases were dried with $\mathrm{MgSO}_{4}$ and a solvent was removed under reduced pressure. The residue was dissolved in chloroform $(30 \mathrm{~mL})$ and manganese dioxide $(0.5 \mathrm{~g})$ was added. The resulting mixture was stirred for 3.5 hours at room temperature, manganese dioxide was filtered off and washed with chloroform $(40 \mathrm{~mL})$. The filtrate was concentrated in vacuo, and the residue was purified by chromatography with a mixture of chloroform - methanol (50:1) as eluent. Pale yellow crystals, yield $1.1 \mathrm{~g}(64 \%) ; \mathrm{mp} 82-84{ }^{\circ} \mathrm{C}$ (from hexane); IR $\left(v_{\max }, \mathrm{cm}^{-1}\right)$ : 2958, $1581(\mathrm{C}=\mathrm{N}), 1450,1243$, 1172, 1156, 821, 765, 723, 707, 601. ${ }^{1} \mathrm{H}$ NMR $\left(400.13 \mathrm{MHz}, \mathrm{CDCl}_{3}\right): \delta 0.28\left(\mathrm{~s}, 3 \mathrm{H}, \mathrm{C}_{3}\right), 1.01$ (s, $\left.3 \mathrm{H}, \underline{\mathrm{C}}_{3}\right), 1.48\left(\mathrm{~s}, 3 \mathrm{H}, \underline{\mathrm{C}}_{3}\right), 1.80$ (dd, AB-system, $\left.2 \mathrm{H}, J_{H, H}^{2} 13.4 \mathrm{~Hz}, \mathrm{C}\left(\mathrm{CH}_{3}\right)_{2} \mathrm{C}_{2} \underline{\mathrm{H}}_{2}\right), 2.89$ (dd, $2 \mathrm{H}, \mathrm{AB}$-system, $\left.J_{H, H}^{2} 13.8 \mathrm{~Hz}, \mathrm{PhC}_{2}\right), 6.42(\mathrm{~s}, 1 \mathrm{H}, \mathrm{N}=\mathrm{C} \underline{\mathrm{H}}), 7.09-7.20(\mathrm{~m}, 5 \mathrm{H}, \mathrm{Ph}) .{ }^{13} \mathrm{C} \mathrm{NMR}$ $\left(100.61 \mathrm{MHz}, \mathrm{CDCl}_{3}\right): \delta 26.8\left(\underline{\mathrm{CH}}_{3}\right), 27.9\left(\underline{\mathrm{CH}}_{3}\right), 29.3\left(\underline{\mathrm{CH}}_{3}\right), 37.7\left(\underline{\mathrm{C}}\left(\mathrm{CH}_{3}\right)_{2}\right), 43.2\left(\mathrm{Ph}^{2} \mathrm{H}_{2}\right), 43.6$ $\left(\underline{\mathrm{CH}}_{2} \mathrm{C}\left(\mathrm{CH}_{3}\right)_{2}\right), 78.1\left(\underline{\mathrm{C}}\left(\mathrm{CH}_{3}\right) \mathrm{CH}_{2} \mathrm{Ph}\right), 126.9,128.3,130.3,136.3(\mathrm{Ph}), 142.1(\underline{\mathrm{CH}}=\mathrm{N})$. Calcd. for $\mathrm{C}_{14} \mathrm{H}_{19} \mathrm{NO}: m / z 217.1461[\mathrm{M}]^{+}$. Found: $m / z$ 217.1462 [M] $]^{+}$.

2,4,4-Trimethyl-2-(naphthalen-1-ylmethyl)-3,4-dihydro-2 $\mathrm{H}$-pyrrole 1-oxide $\mathbf{1 3 b}$ was synthesized according to the procedure described for 13a from magnesium $(0.236 \mathrm{~g}, 9.84 \mathrm{mmol})$, 1-chloromethylnaphthalene $(1.76 \mathrm{~g}, 9.50 \mathrm{mmol})$ and $11(1.0 \mathrm{~g}, 7.87 \mathrm{mmol})$. White powder, yield $1.26 \mathrm{~g}(60 \%) ; \mathrm{mp} 79-82{ }^{\circ} \mathrm{C}$ (from hexane); IR $\left(v_{\max }, \mathrm{cm}^{-1}\right): 3026,2966,1581(\mathrm{C}=\mathrm{N}), 1453$, 1262, 1169, 811, 787, 605. ${ }^{1} \mathrm{H}$ NMR (400.13 MHz, $\mathrm{CDCl}_{3}$ ): $\delta 0.02$ (s, 3H, $\underline{\mathrm{C}}_{3}$ ), 0.95 (s, 3H, $\left.\mathrm{C}_{3}\right), 1.60$ (s, $\left.3 \mathrm{H}, \underline{\mathrm{C}}_{3}\right), 1.75$ (dd, AB-system, $\left.2 \mathrm{H}, J_{H, H}^{2} 13.4 \mathrm{~Hz}, \mathrm{C}\left(\mathrm{CH}_{3}\right)_{2} \underline{\mathrm{C}}_{2}\right), 3.47$ (dd, ABsystem, $2 \mathrm{H}, J^{2}{ }_{H, H} 14.4 \mathrm{~Hz}$, arom- $\left.\underline{\mathrm{H}}_{2}\right), 6.46(\mathrm{~s}, 1 \mathrm{H}, \mathrm{N}=\mathrm{C} \underline{\mathrm{H}}), 7.30-7.39(\mathrm{~m}, 2 \mathrm{H}), 7.41-7.46(\mathrm{~m}$, $2 \mathrm{H}), 7.66\left(\mathrm{~d}, 1 \mathrm{H}, J_{H, H}^{3} 8.1 \mathrm{~Hz}\right), 7.74\left(\mathrm{~d}, 1 \mathrm{H}, J_{H, H}^{3} 8.1 \mathrm{~Hz}\right), 7.98\left(\mathrm{~d}, 1 \mathrm{H}, J_{H, H}^{3} 8.5 \mathrm{~Hz}\right.$, arom.) ${ }^{13} \mathrm{C}$ 
NMR (100.61 MHz, $\left.\left.\mathrm{CDCl}_{3}\right): \delta 26.7\left(\underline{\mathrm{CH}}_{3}\right), 28.4(\underline{\mathrm{CH}})_{3}\right), 29.2\left(\underline{\mathrm{C}} \mathrm{H}_{3}\right), 37.8\left(\mathrm{Napht}-\underline{\mathrm{CH}}_{2}\right), 37.8$ $\left(\underline{\mathrm{C}}\left(\mathrm{CH}_{3}\right)_{2}\right), 43.8\left(\underline{\mathrm{C}} \mathrm{H}_{2} \mathrm{C}\left(\mathrm{CH}_{3}\right)_{2}\right), 79.0\left(\underline{\mathrm{C}}\left(\mathrm{CH}_{3}\right) \mathrm{CH}_{2}-\mathrm{Napht}\right), 123.7,125.3,125.6,126.1,127.6$, 128.5, 128.8, 132.8, 132.9, 133.7 (Napht.),142.0 ( $\underline{\mathrm{CH}}=\mathrm{N})$. Calcd. for $\mathrm{C}_{18} \mathrm{H}_{21} \mathrm{NO}: \mathrm{m} / z 267.1618$ $[\mathrm{M}]^{+}$. Found: $m / z 267.1619[\mathrm{M}]^{+}$. Absorption maximum $-283 \mathrm{~nm}$, emission maximum -337 $\mathrm{nm}$.

(E)-3,3-Dimethyl-5-(2-(naphthalen-1-yl)vinyl)-3,4-dihydro-2 $H$-pyrrole 1-oxide 14a. To a stirring solution of sodium methylate $(0.85 \mathrm{~g}, 15.75 \mathrm{mmol})$ in anhydrous methanol $(7.5 \mathrm{~mL})$ compound 11 (1.0 g, $7.87 \mathrm{mmol})$ and 1-naphthaldehyde (1.84 g, $11.81 \mathrm{mmol})$ were added. Resulting mixture was refluxed for 3.5 hours and cooled. The reaction mixture was diluted with water $(10 \mathrm{~mL})$ and extracted with chloroform $(3 \times 20 \mathrm{~mL})$. Combined organic extract was dried with $\mathrm{MgSO}_{4}$ and the solvent was removed under reduced pressure. The residue was purified by chromatography with a mixture of chloroform - methanol (50:1) as eluent. Pale yellow crystals, yield $1.69 \mathrm{~g}(81 \%)$; mp $113-115^{\circ} \mathrm{C}$ (from mixture hexane - ethyl acetate 5:1); IR $\left(v_{\max }, \mathrm{cm}^{-1}\right)$ : 2955, $1539(\mathrm{C}=\mathrm{N}), 1406,1285,1260,1224,1162,981,798,781,661 .{ }^{1} \mathrm{H}$ NMR $(400.13 \mathrm{MHz}$, $\left.\mathrm{CDCl}_{3}\right): \delta 1.26\left(\mathrm{~s}, 6 \mathrm{H}, \mathrm{C}\left(\mathrm{C}_{3}\right)_{2}\right), 2.81\left(\mathrm{~s}, 2 \mathrm{H}, \mathrm{CH}_{2} \mathrm{C}\left(\mathrm{CH}_{3}\right)_{2}\right), 3.85$ (s, 2H, N=C-C $\left.\underline{\mathrm{H}}_{2}\right), 7.42-7.53$

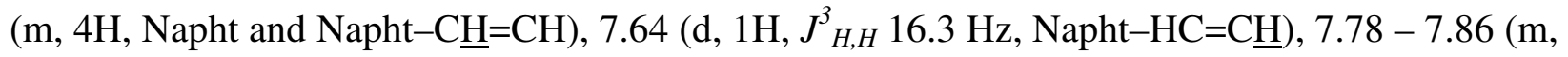

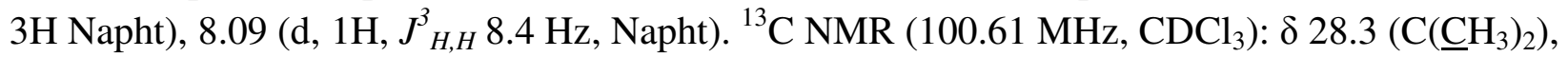
$32.9\left(\underline{\mathrm{C}}\left(\mathrm{CH}_{3}\right)_{2}\right), 43.7\left(\mathrm{~N}=\mathrm{C}-\underline{\mathrm{C}} \mathrm{H}_{2}\right), 75.3\left(\underline{\mathrm{CH}}_{2} \mathrm{C}\left(\mathrm{CH}_{3}\right)_{2}\right), 118.4(\mathrm{Napht}-\mathrm{HC}=\underline{\mathrm{CH}}), 123.0,124.5$, 125.8, 126.1, 126.6, 128.9, 129.6, 131.4 (Napht), 132.8 (Napht-HC=CH), 133.0, 133.8 (Napht), $143.9(\underline{\mathrm{C}}=\mathrm{N})$. Calcd. for $\mathrm{C}_{18} \mathrm{H}_{19} \mathrm{NO}: m / z, 265.1461[\mathrm{M}]^{+}$. Found: $m / z$ 265.1463 [M] $]^{+}$. Absorption maximum - $301 \mathrm{~nm}$, emission maximum - $366 \mathrm{~nm}$.

Crystallographic data for comp 14a. $\mathrm{C}_{18} \mathrm{H}_{19} \mathrm{NO}, M=265.34$, orthorhombic, $P 2_{1} 2_{1} 2_{1}, a=$ 6.3974(2), $b=11.7839(5), c=19.2374(8) \AA, V=1450.2(1) \AA^{3}, Z=4, D_{\text {calcd }}=1.215 \mathrm{~g} \cdot \mathrm{cm}^{-3}$, $\mu(\mathrm{Mo}-K \alpha)=0.075 \mathrm{~mm}^{-1}, \mathrm{~F}(000)=568,\left(\theta 2.03-27.89^{\circ}\right.$, completeness $\left.100 \%\right), \mathrm{T} 200(2) \mathrm{K}$, colorless plate, $(1.00 \times 0.60 \times 0.20) \mathrm{mm}^{3}$, transmission $0.9026-0.9103,30333$ measured reflections in index range $-8<=\mathrm{h}<=7,-15<=\mathrm{k}<=15,-25<=\mathrm{l}<=25,3473$ independent $\left(R_{\text {int }}=\right.$ 0.0452 ), 183 parameters, 3255 observed $[I>2 \sigma(I)], R_{1}=0.0314, w R_{2}=0.1085$ (all data), GOOF 1.153 , largest diff. peak and hole 0.205 and -0.234 e. $\mathrm{A}^{-3}$.

(E)-5-(2-(4-Methoxynaphthalen-1-yl)vinyl)-3,3-dimethyl-3,4-dihydro-2H-pyrrole 1-oxide 14b was synthesized according to the procedure described for 14a from 4-methoxy-1naphthaldehyde $(2.2 \mathrm{~g}, 11.81 \mathrm{mmol})$ and $11(1.0 \mathrm{~g}, 7.87 \mathrm{mmol})$. Yellow crystals, yield $2.00 \mathrm{~g}$ (86\%); mp $154^{\circ} \mathrm{C}$ (from mixture hexane - ethyl acetate 4:1); IR $\left(v_{\max }, \mathrm{cm}^{-1}\right): 2955,1578(\mathrm{C}=\mathrm{N})$, 1545, 1463, 1402, 1373, 1281, 1229, 1096, 963, 766. ${ }^{1} \mathrm{H}$ NMR (400.13 MHz, $\left.\mathrm{CDCl}_{3}\right): \delta 1.24$ (s, $\left.6 \mathrm{H}, \mathrm{C}\left(\mathrm{C}_{3}\right)_{2}\right), 2.77$ (s, 2H, $\left.\underline{\mathrm{H}}_{2} \mathrm{C}=\mathrm{N}\right), 3.83$ (s, 2H, $\left.\underline{\mathrm{H}}_{2} \mathrm{~N}=\mathrm{C}\right), 3.97$ (s, 3H, $\left.\mathrm{C}_{3} \mathrm{O}\right), 6.79(\mathrm{~d}, 1 \mathrm{H}$, $J_{H, H}^{3} 8.2 \mathrm{~Hz}$, arom.), $7.39\left(\mathrm{~d}, 1 \mathrm{H}, J_{H, H}^{3} 16.2 \mathrm{~Hz}\right.$, arom-CH=CH$), 7.43-7.488$ (m, 1H, arom.), $7.50-7.56(\mathrm{~m}, 2 \mathrm{H}$, arom. and arom- $\underline{\mathrm{H}}=\mathrm{CH}), 7.81\left(\mathrm{~d}, 1 \mathrm{H}, J_{H, H}^{3} 8.2 \mathrm{~Hz}\right.$, arom.), $8.01-8.05$ (m, $1 \mathrm{H}), 8.25-8.28\left(\mathrm{~m}, 1 \mathrm{H}\right.$, arom). ${ }^{13} \mathrm{C} \mathrm{NMR}\left(100.61 \mathrm{MHz}, \mathrm{CDCl}_{3}\right): \delta 28.3\left(\mathrm{C}\left(\mathrm{CH}_{3}\right)_{2}\right), 32.9$ $\left(\underline{\mathrm{C}}\left(\mathrm{CH}_{3}\right)_{2}\right), 43.6\left(\underline{\mathrm{CH}}_{2} \mathrm{C}=\mathrm{N}\right), 55.7\left(\underline{\mathrm{CH}}_{3} \mathrm{O}\right), 75.1\left(\underline{\mathrm{CH}}_{2} \mathrm{~N}=\mathrm{C}\right), 104.1,116.1,122.6$ (arom), 122.8 ( $\underline{\mathrm{CH}}=\mathrm{CH}$-arom), 125.2 (arom), 125.4 (CH=ㅡH-arom), 125.6, 127.1, 132.0, 132.8 (arom), 144.3 
$(\underline{\mathrm{C}}=\mathrm{N}), 156.6$ (arom). Calcd. for $\mathrm{C}_{19} \mathrm{H}_{21} \mathrm{NO}_{2}$ (295.16): C 77.26, $\mathrm{H}$ 7.17, $\mathrm{N}$ 4.74. Found: C 77.32, $\mathrm{H}$ 6.97, N 4.68. Absorption maximum - $324 \mathrm{~nm}$, emission maximum - $413 \mathrm{~nm}$.

(E)-5-(2-(Anthracen-9-yl)vinyl)-3,3-dimethyl-3,4-dihydro-2H-pyrrole 1-oxide 14c was synthesized according to the procedure described for 14a from anthracene-9-carbaldehyde (2.43 $\mathrm{g}, 11.81 \mathrm{mmol}$ ) and 11 (1.0 g, $7.87 \mathrm{mmol})$. Orange crystals, yield $1.81 \mathrm{~g}(73 \%)$; $\mathrm{mp} 191{ }^{\circ} \mathrm{C}$ (from ethyl acetate); IR $\left(v_{\max }, \mathrm{cm}^{-1}\right): 3043,2959,1540(\mathrm{C}=\mathrm{N}), 1444,1284,1272,1230,1213,1174$, 1160, 891, 850, 739. ${ }^{1} \mathrm{H}$ NMR (400.13 MHz, $\left.\mathrm{CDCl}_{3}\right): \delta 1.33$ (s, 6H, $\left.\mathrm{C}\left(\mathrm{CH}_{3}\right)_{2}\right), 2.92$ (s, 2H, $\left.\mathrm{C}_{2} \mathrm{C}=\mathrm{N}\right), 3.93\left(\mathrm{~s}, 2 \mathrm{H}, \underline{\mathrm{C}}_{2} \mathrm{~N}=\mathrm{C}\right), 7.32\left(\mathrm{~d}, 1 \mathrm{H}, J_{H, H}^{3} 16.7 \mathrm{~Hz}\right.$, arom-CH=C $\left.\underline{\mathrm{H}}\right), 7.41-7.48(\mathrm{~m}, 4 \mathrm{H}$, arom.), $7.76\left(\mathrm{~d}, 1 \mathrm{H}, J_{H, H}^{3} 16.7 \mathrm{~Hz}\right.$, arom- $\left.\underline{\mathrm{H}}=\mathrm{CH}\right), 7.93-7.97$ (m, 2H.), 8.20-8.24 (m, 2H), 8.36 (s, 1H, arom.). ${ }^{13} \mathrm{C} \mathrm{NMR}\left(100.61 \mathrm{MHz}, \mathrm{CDCl}_{3}\right): \delta 28.5\left(\mathrm{C}(\underline{\mathrm{CH}})_{2}\right), 33.0\left(\underline{\mathrm{C}}\left(\mathrm{CH}_{3}\right)_{2}\right), 43.8$ $\left(\underline{\mathrm{CH}}_{2} \mathrm{C}=\mathrm{N}\right), 75.4\left(\underline{\mathrm{CH}}_{2} \mathrm{~N}=\mathrm{C}\right), 124.7$ (arom-CH=ㅡㅐ), $125.5(4 \mathrm{C}), 126.2(2 \mathrm{C}), 128.0,128.9$ (2C), 129.4 (2C), 130.6, 131.4 (2C, arom.), 133.6 (arom- $\underline{\mathrm{CH}}=\mathrm{CH}), 144.2(\underline{\mathrm{C}}=\mathrm{N})$. Calcd. for $\mathrm{C}_{22} \mathrm{H}_{21} \mathrm{NO}$ (295.16): C 83.78, H 6.71, N 4.44. Found: C 83.42, H 6.66, N 4.58. Absorption maximum - 371 $\mathrm{nm}$, emission maximum $-447 \mathrm{~nm}$.

(E)-3,3-Dimethyl-5-(2-(1-methyl-2-phenyl-1H-indol-3-yl)vinyl)-3,4-dihydro-2H-pyrrole 1oxide 14d was synthesized according to the procedure described for 14a from 1-methyl-2phenyl-1H-indole-3-carbaldehyde $(2.78 \mathrm{~g}, 11.81 \mathrm{mmol})$ and 11 (1.0 g, $7.87 \mathrm{mmol})$. Yellow crystals, yield $0.70 \mathrm{~g}(26 \%)$; mp $212-215^{\circ} \mathrm{C}$ (from mixture hexane - ethyl acetate 5:1 with decomposition); IR $\left(v_{\max }, \mathrm{cm}^{-1}\right): 2925,1606(\mathrm{C}=\mathrm{N}), 1549,1470,1440,1384,1281,1227,1157$, 1069, 957, 822, 739, 698. ${ }^{1} \mathrm{H}$ NMR $\left.\left(300.13 \mathrm{MHz}^{\mathrm{CDCl}}\right)_{3}\right): \delta 1.16\left(\mathrm{~s}, 6 \mathrm{H}, \mathrm{C}\left(\mathrm{CH}_{3}\right)_{2}\right), 2.49$ (s, 2H, $\left.\mathrm{C}_{2} \mathrm{C}=\mathrm{N}\right), 3.56\left(\mathrm{~s}, 3 \mathrm{H}, \mathrm{NC}_{3}\right), 3.76\left(\mathrm{~s}, 2 \mathrm{H}, \underline{\mathrm{CH}}_{2} \mathrm{~N}=\mathrm{C}\right), 6.78\left(\mathrm{~d}, 1 \mathrm{H}, J_{H, H}^{3} 16.7 \mathrm{~Hz}, \mathrm{CH}=\mathrm{C} \underline{\mathrm{H}}-\right.$ $\mathrm{C}=\mathrm{N}), 7.24-7.29(\mathrm{~m}, 2 \mathrm{H}), 7.30-7.32(\mathrm{~m}, 1 \mathrm{H}$, arom), $7.34-7.38(\mathrm{~m}, 3 \mathrm{H}$, arom and $\mathrm{CH}=\mathrm{CH}-$ $\mathrm{C}=\mathrm{N}), 7.42-7.52(\mathrm{~m}, 3 \mathrm{H}), 8.15-8.19\left(\mathrm{~m}, 1 \mathrm{H}\right.$, arom). ${ }^{13} \mathrm{C} \mathrm{NMR}\left(75.47 \mathrm{MHz}, \mathrm{CDCl}_{3}\right): \delta 28.3$

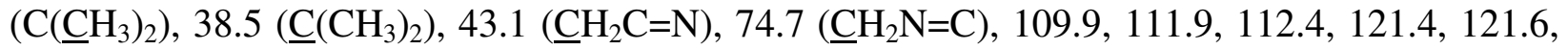
123.1, 125.2, 128.6 (2C, arom), $129.0(\mathrm{CH}=\underline{\mathrm{CH}}-\mathrm{C}=\mathrm{N}), 130.5,130.9$ (2C, arom.), 131.0 $(\underline{\mathrm{CH}}=\mathrm{CH}-\mathrm{C}=\mathrm{N}), 138.0,143.4$ (arom.), $145.3(\mathrm{C}=\mathrm{N})$. Calcd. for $\mathrm{C}_{23} \mathrm{H}_{24} \mathrm{~N}_{2} \mathrm{O}: m / z 344.1883[\mathrm{M}]^{+}$. Found: $m / z 344.1880[\mathrm{M}]^{+}$. Absorption maximum - $256 \mathrm{~nm}$, emission maximum $-383 \mathrm{~nm}$.

Crystallographic data for comp 14d. $\mathrm{C}_{23} \mathrm{H}_{24} \mathrm{~N}_{2} \mathrm{O}, M=344.44$, orthorhombic, Pbca, $a=$ $15.4643(6), b=14.2594(6), c=17.2945(8) \AA, V=3813.6(3) \AA^{3}, Z=8, D_{\text {calcd }}=1.200 \mathrm{~g} \cdot \mathrm{cm}^{-3}$, $\mu(\mathrm{Mo}-\mathrm{K \alpha})=0.074 \mathrm{~mm}^{-1}, \mathrm{~F}(000)=1472,\left(\theta 2.27-27.53^{\circ}\right.$, completeness 99.5\%), $\mathrm{T} 296(2) \mathrm{K}$, yellow pyramid, $(0.80 \times 0.60 \times 0.60) \mathrm{mm}^{3}$, transmission $0.8912-0.9103,71876$ measured reflections in index range $-20<=\mathrm{h}<=20,-18<=\mathrm{k}<=18,-22<=\mathrm{l}<=22,4379$ independent $\left(R_{\text {int }}=\right.$ 0.0412 ), 238 parameters, 3480 observed $[I>2 \sigma(I)], R_{1}=0.0494, w R_{2}=0.1415$ (all data), GOOF 1.052 , largest diff. peak and hole 0.205 and -0.234 e. $\mathrm{A}^{-3}$.

The naphthyl fragment in the molecule of compounds 5a and 14a is planar within $\pm 0.013(1)$ and $\pm 0.027(1)$ with bond lengths equal within the experimental errors and being close to average statistical values ${ }^{21}$. The indole fragment in the molecule of compound $\mathbf{1 4 d}$ is planar within $\pm 0.008(1) \AA$. The parameters of intramolecular hydrogen bond $\mathrm{C} 13-\mathrm{H} \ldots \mathrm{N} 2$ of 5a are H...N 2.59, C...N 3.075(4) $\AA$, C-H...N $112^{\circ}$ (Wan der Waals radius sums $\operatorname{are}^{22}$ H...O 2.68, C...O 3.35, H...N 2.74 and C...N 3.41 $\AA$ ). Supramolecular structure of 5a crystal can be described as 
$\pi(\mathrm{C} 9 \div \mathrm{C} 14) \ldots \pi(\mathrm{C} 9 \div \mathrm{C} 14)$-stacking dimmers (with inter-plane and inter-centroid distances being equal to 3.3698(5) and 3.5389(7) $\AA$ respectively) combined into layers being parallel to $(b, c)$ plane by $\mathrm{C} 12-\mathrm{H} . . . \pi(\mathrm{C} 5 \mathrm{C} 6 \mathrm{C} 7 \mathrm{C} 8 \mathrm{C} 9 \mathrm{C} 14)$ interactoin (with distances from $\mathrm{H}$ to cycle plain and center equal to 2.98 and $3.10 \AA$ ) and $\mathrm{C} 3-\mathrm{H}$...O3 interactions (with H...O 2.30, C...O 3.141(2) and $\mathrm{C}-\mathrm{H} . . . \mathrm{O} 142^{\circ}$ )

Torsion angle $\mathrm{C} 8=\mathrm{C} 9-\mathrm{C} 10-\mathrm{C} 11$ of $\mathbf{1 4 d}$ equaling to $33.7(2)^{\circ}$ indicates decreasing of $\mathrm{C} 8=\mathrm{C} 9$ bond conjugation with naphthyl fragment which leads to lengthening of C9-C10 bond to 1.474(2) $\AA$ in comparison with $1.445(2)$ for the same bond of $\mathbf{1 4 d}$ (torsion angle $\mathrm{C} 8=\mathrm{C} 9-\mathrm{C} 10-\mathrm{C} 11$ is $\left.179.4(1)^{\circ}\right)$. The dihydropyrrole cycle in molecules of the compounds $\mathbf{1 4 a}$ and $\mathbf{1 4 d}$ adopts the envelope conformation with a puckering angle equal $25.8(1)$ and $25.5(2)^{\circ}$. The conjugation of double bonds $\mathrm{N} 1=\mathrm{C} 5$ of dihydropyrrole cicle and double bonds $\mathrm{C} 8=\mathrm{C} 9$ in both compounds are indicated by values of $\mathrm{C} 5-\mathrm{C} 8$ and $\mathrm{C} 5=\mathrm{N} 1$ bond lengths $(1.437(2)$ and 1.431(2) $\AA$ for the first bond, 1.316(2) and 1.312(2) $\AA$ for the second one), the torsion angle $\mathrm{N} 1=\mathrm{C} 5-\mathrm{C} 8=\mathrm{C} 9$ is $165.0(1)^{\circ}$ for $14 \mathbf{a}$ and $178.0(1)^{\circ}$ for $14 d$. Moreover, the averaged length of unconjugated $\mathrm{C} 5=\mathrm{N} 1$ bond on the set of 21 compound structures with 3,4-dihydropyrroline 1-oxide fragment from $\mathrm{CSDB}^{23}$ equals to1.289(5) $\AA$. Angle between indole and phenyl fragment planes for $\mathbf{1 4 d}$ is $61.7(1)^{\circ}$. The nitroxyl group of both compounds forms a big number of intermolecular hydrogen bonds (table 1) characterizing together with $\mathrm{C}-\mathrm{H} \quad \pi$ interactions cryctal structure of compounds 14a and 14d. C6-H $\pi(\mathrm{C} 10 \mathrm{C} 11 \mathrm{C} 12 \mathrm{C} 13 \mathrm{C} 14 \mathrm{C} 19)$ interaction of compound $14 \mathbf{a}$ is described by distances from hydrogen atom to plain and center of cycle equaling to 2.89 and $2.98 \AA, \mathrm{C} 2-\mathrm{H}$ $\pi(\mathrm{C} 10 \div \mathrm{C} 14), \mathrm{C} 4-\mathrm{H} \pi(\mathrm{C} 13 \div \mathrm{C} 18), \mathrm{C} 6-\mathrm{H} \ldots \pi(\mathrm{C} 20 \div \mathrm{C} 25)$, interactions of compounds $14 \mathrm{~d}$ have distances from atom $\mathrm{H}$ to the plains $2.87,2.93,2.82 \AA$ and to the centers of cycles $-2.95,2.95$, $2.87 \AA$.

(E)-2,4,4-Trimethyl-2-(2-(naphthalen-1-yl)vinyl)pyrrolidin-1-ol 15a. To a stirred solution of methyllithium, prepared from lithium $(0.143 \mathrm{~g}, 20.4 \mathrm{mmol})$ and methyl iodide $(0.63 \mathrm{~g}, 10.2$ $\mathrm{mmol})$ in anhydrous ether $(9 \mathrm{~mL})$, a solution of $\mathbf{1 4 a}(0.9 \mathrm{~g}, 3.4 \mathrm{mmol})$ in anhydrous THF $(3 \mathrm{~mL})$ was added. The resulting mixture was stirred for 2 hours at room temperature. The excess of methyllithium was decomposed by addition of water $(10 \mathrm{~mL})$. Ether solution was separated and water layer was extracted with chloroform $(3 \times 20 \mathrm{~mL})$. Combined organic phases were dried with $\mathrm{MgSO}_{4}$ and the solvent was removed under reduced pressure. The product was precipitated by addition of ether to residue, filtered off and dried. White powder, yield $0.85 \mathrm{~g}(89 \%)$; IR ( $v_{\max }$, $\left.\mathrm{cm}^{-1}\right): 3227(\mathrm{OH}), 2964,2925,2854,1445,1169,1082,980,792,779 .{ }^{1} \mathrm{H}$ NMR $(400.13 \mathrm{MHz}$, $\left.\mathrm{CDCl}_{3}\right): \delta 1.13\left(\mathrm{~s}, 6 \mathrm{H}, \mathrm{C}\left(\mathrm{CH}_{3}\right)_{2}\right), 1.45\left(\mathrm{~s}, 3 \mathrm{H}, \mathrm{CH}_{3}\right), 1.84$ (dd, AB-system, $2 \mathrm{H}, J_{H, H}^{2} 13.0 \mathrm{~Hz}$, $\left.\mathrm{C}_{2} \mathrm{C}\left(\mathrm{CH}_{3}\right)_{2}\right), 3.00\left(\mathrm{~s}, 2 \mathrm{H}, \underline{\mathrm{CH}}_{2} \mathrm{NOH}\right), 6.54\left(\mathrm{~d}, 2 \mathrm{H}, J_{H, H}^{3} 15.4 \mathrm{~Hz}, \mathrm{Napht}-\mathrm{CH}=\mathrm{C} \underline{\mathrm{H}}\right), 7.22(\mathrm{~d}, 2 \mathrm{H}$, $\left.J_{H, H}^{3} 15.4 \mathrm{~Hz}, \mathrm{Napht}-\mathrm{C} \underline{\mathrm{H}}=\mathrm{CH}\right), 7.37-7.51(\mathrm{~m}, 4 \mathrm{H}, \mathrm{Napht}$. and $\mathrm{N}-\mathrm{OH}), 7.60\left(\mathrm{~d}, 1 \mathrm{H}, J_{H, H}^{3} 6.1\right.$ $\mathrm{Hz}), 7.73\left(\mathrm{~d}, 1 \mathrm{H}, J_{H, H}^{3} 7.6 \mathrm{~Hz}\right), 7.82\left(\mathrm{~d}, 1 \mathrm{H}, J_{H, H}^{3} 6.9 \mathrm{~Hz}\right), 8.09\left(\mathrm{~d}, 1 \mathrm{H}, J_{H, H}^{3} 7.3 \mathrm{~Hz}, \mathrm{Napht}.\right){ }^{13} \mathrm{C}$ NMR (75.47 MHz, $\left.\mathrm{CDCl}_{3}\right): \delta 30.9\left(\mathrm{C}\left(\underline{\mathrm{CH}}_{3}\right)_{2}\right), 31.1\left(\underline{\mathrm{CH}}_{3}\right), 33.2\left(\underline{\mathrm{C}}\left(\mathrm{CH}_{3}\right)_{2}\right), 50.9\left(\underline{\mathrm{CH}}_{2} \mathrm{C}\left(\mathrm{CH}_{3}\right)_{2}\right)$, $68.5\left(\mathrm{CH}_{2} \mathrm{NOH}\right), 69.1\left(\underline{\mathrm{CCH}}_{3}\right), 123.9,124.0,125.8$ (2C), 126.0 (Napht.), 126.2 (Napht $\mathrm{CH}=\underline{\mathrm{CH}}$ ), 127.7, 128.6, 131.4, 133.7, 135.4 (Napht), 138.4 (Napht- $\underline{\mathrm{CH}}=\mathrm{CH})$. Calcd. for $\mathrm{C}_{19} \mathrm{H}_{23} \mathrm{NO}: m / z 281.1774[\mathrm{M}]^{+}$. Found: $m / z$ 281.1773 [M] $]^{+}$. 
(E)-2,4,4-Trimethyl-2-(2-(naphthalen-1-yl)vinyl)-3,4-dihydro-2H-pyrrole 1-oxide 16 a. Manganese dioxide $(100 \mathrm{mg})$ was added to a stirred solution of $15 \mathbf{a}(0.08 \mathrm{~g}, 0.28 \mathrm{mmol})$ in THF $(5 \mathrm{~mL})$. The resulting mixture was stirred at room temperature for 0.5 hours, then manganese dioxide was filtered off and washed with chloroform $(10 \mathrm{~mL})$. Filtrate was concentrated in vacuo, product was purified by preparative TLC on alumina with chloroform as eluent. Colorless crystals, yield $0.065 \mathrm{~g}(82 \%)$; mp $134-136^{\circ} \mathrm{C}$ (from mixture hexane - ethyl acetate 4:1); IR $\left(v_{\max }, \mathrm{cm}^{-1}\right): 3043,2960,1580(\mathrm{C}=\mathrm{N}), 1242,1163,959,802,791 .{ }^{1} \mathrm{H}$ NMR $(300.13 \mathrm{MHz}$, $\left.\mathrm{CDCl}_{3}\right): \delta 1.26\left(\mathrm{~s}, 6 \mathrm{H}, \mathrm{C}\left(\mathrm{CH}_{3}\right)_{2}\right), 1.74\left(\mathrm{~s}, 3 \mathrm{H}, \mathrm{C}_{3}\right), 2.28\left(\mathrm{dd}, \mathrm{AB}\right.$-system, $2 \mathrm{H}, J_{H, H}^{2} 13.2 \mathrm{~Hz}$,

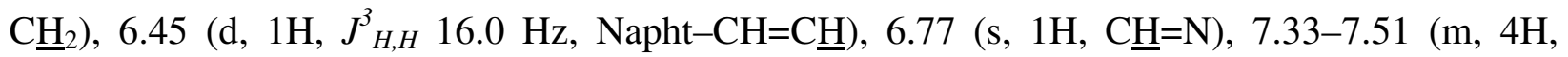

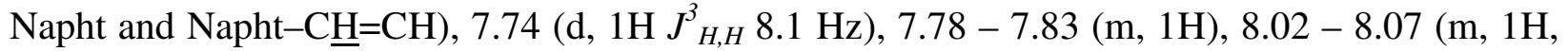
Napht.). ${ }^{13} \mathrm{C}$ NMR $\left(75.47 \mathrm{MHz}, \mathrm{CDCl}_{3}\right): \delta 26.4\left(\mathrm{C}_{3}\right), 28.0\left(\mathrm{C}\left(\underline{\mathrm{CH}}_{3}\right)_{2}\right), 28.5\left(\mathrm{C}\left(\underline{\mathrm{CH}}_{3}\right)_{2}\right), 38.8$ $\left.\left(\underline{\mathrm{C}}\left(\mathrm{CH}_{3}\right)_{2}\right), 48.8\left(\underline{\mathrm{CH}}_{2}\right), 78.4(\mathrm{CH}=\mathrm{N}-\underline{\mathrm{C}}), 123.8,124.2,125.6,125.9\right), 126.2$ (Napht.), 127.3 (Napht-CH=프), 128.3, 128.6, 131.3, 133.6, 134.1 (Napht.), 134.9 (Napht- $\underline{\mathrm{C}} \mathrm{H}=\mathrm{CH}), 141.3$ $(\underline{\mathrm{CH}}=\mathrm{N})$. Calcd. for $\mathrm{C}_{19} \mathrm{H}_{21} \mathrm{NO}: \mathrm{m} / z 279.1618[\mathrm{M}]^{+}$. Found: $\mathrm{m} / z 279.1614[\mathrm{M}]^{+}$. Absorption maximum $-300 \mathrm{~nm}$, emission maximum $-359 \mathrm{~nm}$.

(E)-2-(2-(4-Methoxynaphthalen-1-yl)vinyl)-2,4,4-trimethyl-3,4-dihydro-2H-pyrrole 1-oxide 16b. To a stirred solution of methyllithium, prepared from lithium $(0.200 \mathrm{~g}, 28.47 \mathrm{mmol})$ and methyl iodide $(0.89 \mathrm{~g}, 14.24 \mathrm{mmol})$ in anhydrous ether $(10 \mathrm{~mL}))$ a solution of compound 14b $(1.4 \mathrm{~g}, 4.75 \mathrm{mmol})$ in anhydrous THF $(15 \mathrm{~mL})$ was added dropwise. A resulted mixture was stirred for 2 hours at room temperature, then an excess of methyllithium was decomposed by addition of water $(10 \mathrm{~mL})$. Ether solution was separated and water layer was extracted with chloroform $(4 \times 20 \mathrm{~mL})$. Combined organic phases were dried with $\mathrm{MgSO}_{4}$ and the solvent was removed under reduced pressure. The residue was dissolved in chloroform $(30 \mathrm{~mL})$ and manganese dioxide $(0.2 \mathrm{~g})$ was added. The resulting mixture was stirred for 1 hour at room temperature, then manganese dioxide was filtered off and washed with chloroform $(40 \mathrm{~mL})$. Filtrate was concentrated in vacuo, and the product was purified by chromatography with a mixture of chloroform - methanol (50:1) as eluent. Pale yellow crystals, yield $1.17 \mathrm{~g} \mathrm{(80 \% );} \mathrm{mp}$ $116^{\circ} \mathrm{C}$ (from mixture hexane - ethyl acetate 5:1); IR $\left(v_{\max }, \mathrm{cm}^{-1}\right): 2964,1575(\mathrm{C}=\mathrm{N}), 1462$, 1388, 1271, 1244, 1164, 1095, 821, 766. ${ }^{1} \mathrm{H}$ NMR $\left(300.13 \mathrm{MHz}, \mathrm{CDCl}_{3}\right): \delta 1.25(\mathrm{~s}, 6 \mathrm{H}$, $\left.\mathrm{C}\left(\mathrm{C}_{3}\right)_{2}\right), 1.73$ (s, $\left.3 \mathrm{H}, \underline{\mathrm{CH}}_{3}\right), 2.27$ (dd, AB-system, $\left.2 \mathrm{H}, J_{H, H}^{2} 13.3 \mathrm{~Hz}, \mathrm{C}\left(\mathrm{CH}_{3}\right)_{2} \mathrm{C}_{2}\right), 3.96$ (s, $3 \mathrm{H}$, $\left.\mathrm{CH}_{3} \mathrm{O}\right), 6.37\left(\mathrm{~d}, 1 \mathrm{H}, J_{H, H}^{3} 15.9 \mathrm{~Hz}, \mathrm{CH}=\mathrm{CH}-\right.$ arom $), 6.74\left(\mathrm{~d}, 1 \mathrm{H}, J_{H, H}^{3} 8.2 \mathrm{~Hz}\right.$, arom), $6.76(\mathrm{~s}, 1 \mathrm{H}$, $\mathrm{C} \underline{\mathrm{H}}=\mathrm{N}), 7.27\left(\mathrm{~d}, 1 \mathrm{H}, J_{H, H}^{3} 15.9 \mathrm{~Hz}, \mathrm{CH}=\mathrm{C} \underline{\mathrm{H}}-\mathrm{arom}\right), 7.41-7.53(\mathrm{~m}, 3 \mathrm{H}), 7.96-8.01(\mathrm{~m}, 1 \mathrm{H})$, $8.23-8.27$ (m, $1 \mathrm{H}$, arom.). ${ }^{13} \mathrm{C} \mathrm{NMR}\left(75.47 \mathrm{MHz}, \mathrm{CDCl}_{3}\right): \delta 26.5\left(\mathrm{C}\left(\mathrm{C}_{3}\right)_{2}\right), 28.1\left(\mathrm{C}\left(\underline{\mathrm{CH}}_{3}\right)_{2}\right)$, $\left.28.5\left(\underline{\mathrm{CH}_{3}} \mathrm{CCH}=\mathrm{CH}\right), 38.8\left(\underline{\mathrm{C}}\left(\mathrm{CH}_{3}\right)_{2}\right), 48.8(\underline{\mathrm{CH}})_{2}\right), 55.6\left(\underline{\mathrm{CH}}_{3} \mathrm{O}\right), 78.5\left(\mathrm{CH}_{3} \underline{\mathrm{CCH}}=\mathrm{CH}\right), 103.8$, 122.5, 123.5, 124.4, 125.2, 125.5, 126.5, 126.7 (arom.), 127.2 ( $\underline{\mathrm{CH}}=\mathrm{CH}$-arom), 132.1 (arom), $132.9\left(\mathrm{CH}=\underline{\mathrm{CH}}\right.$-arom), $141.3(\underline{\mathrm{CH}}=\mathrm{N}), 155.6$ (arom). Calcd. for $\mathrm{C}_{20} \mathrm{H}_{23} \mathrm{NO}_{2}$ (309.40): $\mathrm{C} 77.64, \mathrm{H}$ 7.49, N 4.53. Found: C 77.20, H 7.51, N 4.58. Absorption maximum - $318 \mathrm{~nm}$, emission maximum $-387 \mathrm{~nm}$.

(E)-2-(2-(Anthracen-9-yl)vinyl)-2,4,4-trimethyl-3,4-dihydro-2H-pyrrole 1-oxide 16c was synthesized according to the procedure, described for $\mathbf{1 6 b}$ from $14 \mathbf{c}(1.40 \mathrm{~g}, 4.75 \mathrm{mmol})$. Yellow 
crystals, yield $0.78 \mathrm{~g}(50 \%)$; mp $135-137^{\circ} \mathrm{C}$ (with decomposition); IR ( $\left.v_{\max }, \mathrm{cm}^{-1}\right)$ : 3022, 2963, 2866, $1574(\mathrm{C}=\mathrm{N}), 1455,1441,1350,1267,1244,1158,992,894,738,608 .{ }^{1} \mathrm{H}$ NMR (400.13 $\left.\mathrm{MHz}, \mathrm{CDCl}_{3}\right): \delta 1.32$ (s, 6H, C( $\left.\left.\mathrm{C}_{3}\right)_{2}\right), 1.89$ (s, 3H, $\left.\underline{\mathrm{CH}}_{3}\right), 2.35$ (dd, AB-system, $2 \mathrm{H}, J_{H, H}^{2} 13.3$ $\left.\mathrm{Hz}, \mathrm{C}\left(\mathrm{CH}_{3}\right)_{2} \mathrm{CH}_{2}\right), 6.22$ (d, $1 \mathrm{H}, J_{H, H}^{3} 16.4 \mathrm{~Hz}, \mathrm{CH}=\mathrm{CH}-$ arom), $6.99(\mathrm{CH}=\mathrm{N}), 7.38-7.48(\mathrm{~m}, 5 \mathrm{H}$, arom and $\mathrm{CH}=\mathrm{CH}$-arom), $7.93-7.97(\mathrm{~m}, 2 \mathrm{H}), 8.20-8.24(\mathrm{~m}, 2 \mathrm{H}), 8.34$ (s, 1H, arom.). ${ }^{13} \mathrm{C}$ NMR (100.61 MHz, $\left.\mathrm{CDCl}_{3}\right): \delta 25.6\left(\mathrm{C}\left(\mathrm{C}_{3}\right)_{2}\right), 28.0\left(\mathrm{C}\left(\underline{\mathrm{C}}_{3}\right)_{2}\right), 28.3\left(\underline{\mathrm{CH}}_{3} \mathrm{CCH}=\mathrm{CH}\right), 39.0$ $\left(\underline{\mathrm{C}}\left(\mathrm{CH}_{3}\right)_{2}\right), 48.7\left(\underline{\mathrm{CH}}_{2}\right), 78.2\left(\mathrm{CH}_{3} \underline{\mathrm{CCH}}=\mathrm{CH}\right), 125.0$ (2C), 125.5 (2C), 126.5 (arom.), 126.7

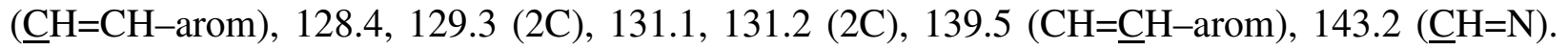
Calcd. for $\mathrm{C}_{23} \mathrm{H}_{23} \mathrm{NO}: \mathrm{m} / z$ z $329.1772[\mathrm{M}]^{+}$. Found: $\mathrm{m} / z$ 329.1774 [M] ${ }^{+}$. Absorption maximum $372 \mathrm{~nm}$, emission maximum - $435 \mathrm{~nm}$.

Hydrogenation of compounds $14 \mathbf{a}-\mathbf{b}(7.55 \mathrm{mmol})$ was carried out in methanol $(20 \mathrm{~mL})$ in the presence of $\mathrm{Pd} / \mathrm{C}(5 \% \mathrm{Pd})$ as catalyst at atmospheric pressure for 4 hours. The catalyst was filtered off, washed with methanol $(40 \mathrm{~mL})$ and the filtrate was concentrated in vacuo, products were isolated by chromatography with a mixture of ethyl acetate - methanol (1:1) as eluent. Compounds 17a-b and 18a-b were eluted sequentially.

3,3-Dimethyl-5-(2-(naphthalen-1-yl)ethyl)-3,4-dihydro-2H-pyrrole 17a. Yellow oil, yield $0.19 \mathrm{~g}(10 \%)$; IR $\left(v_{\max }, \mathrm{cm}^{-1}\right): 2953,2928,2864,1639(\mathrm{C}=\mathrm{N}), 1462,1428,798,780 .{ }^{1} \mathrm{H} \mathrm{NMR}$ $\left(300.13 \mathrm{MHz}, \mathrm{CDCl}_{3}\right): \delta 1.08\left(\mathrm{~s}, 6 \mathrm{H}, \mathrm{C}\left(\mathrm{CH}_{3}\right)_{2}\right), 2.31\left(\mathrm{t}, 2 \mathrm{H}, J_{H, H}^{4} 1.7 \mathrm{~Hz},\left(\mathrm{CH}_{3}\right)_{2} \mathrm{CC} \underline{H}_{2} \mathrm{C}=\mathrm{N}\right)$, 2.67-2.74 (m, 2H, $\underline{\mathrm{C}}_{2}-\mathrm{CH}_{2}-\mathrm{Napht}$ ), 3.35-3.42 (m, 2H, $\mathrm{CH}_{2}-\mathrm{C}_{2}-\mathrm{Napht}$ ), 3.59 (m, 2H, $\left.\left(\mathrm{CH}_{3}\right)_{2} \mathrm{C}-\mathrm{CH}_{2}-\mathrm{N}=\mathrm{C}\right), 7.33-7.41(\mathrm{~m}, 2 \mathrm{H}), 7.43-7.54(\mathrm{~m}, 2 \mathrm{H}), 7.69-7.73(\mathrm{~m}, 1 \mathrm{H}), 7.82-7.86(\mathrm{~m}$, $1 \mathrm{H}), 8.04-8.08$ (m, 1H, Napht). $\left.{ }^{13} \mathrm{C} \mathrm{NMR}\left(75.46 \mathrm{MHz}, \mathrm{CDCl}_{3}\right): \delta 28.1\left(\mathrm{C}_{\left(\mathrm{CH}_{3}\right.}\right)_{2}\right), 29.5$

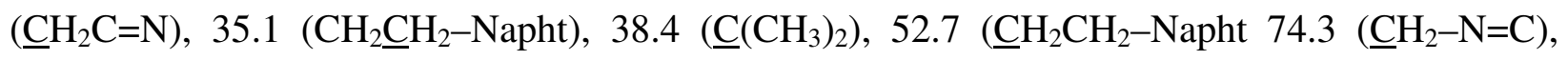
123.7, 125.5, 125.6, 125.8, 126.0, 126.9, 128.8, 131.8, 133.9, 137.7 (Napht), 177.3 ( $\underline{\mathrm{C}}=\mathrm{N})$. Calcd. for $\mathrm{C}_{18} \mathrm{H}_{21} \mathrm{~N}: m / z 251.1669[\mathrm{M}]^{+}$. Found: $m / z$ 250.1588 [M-1] ${ }^{+}$.

3,3-Dimethyl-5-(2-(naphthalen-1-yl)ethyl)-3,4-dihydro-2H-pyrrole 1-oxide 18a. Pale yellow oil, yield $1.61 \mathrm{~g}(80 \%)$; IR $\left(v_{\max }, \mathrm{cm}^{-1}\right): 2958,1625,1597(\mathrm{C}=\mathrm{N}), 1431,1319,1249,1167,800$, 754. ${ }^{1} \mathrm{H}$ NMR (400.13 MHz, $\left.\mathrm{CDCl}_{3}\right): \delta 0.94\left(\mathrm{~s}, 6 \mathrm{H}, \mathrm{C}\left(\mathrm{C}_{3}\right)_{2}\right), 2.11\left(\mathrm{~s}, 2 \mathrm{H}, \mathrm{C}_{2} \mathrm{C}=\mathrm{N}\right), 2.87$ (t, $2 \mathrm{H}, J_{H, H}^{3} 7.5 \mathrm{~Hz}, \underline{\mathrm{C}}_{2} \mathrm{CH}_{2}-\mathrm{Napht}$ ), 3.27 (t, 2H, $J_{H, H}^{3} 7.5 \mathrm{~Hz}, \mathrm{CH}_{2} \underline{\mathrm{C}}_{2}-\mathrm{Napht}$ ), 3.72 (s, 2H, $\left.\mathrm{C}=\mathrm{N}(\mathrm{O})-\mathrm{CH}_{2}\right), 7.28-7.33(\mathrm{~m}, 2 \mathrm{H}), 7.39-7.44(\mathrm{~m}, 1 \mathrm{H}), 7.45-7.50(\mathrm{~m}, 1 \mathrm{H}), 7.63-7.67(\mathrm{~m}, 1 \mathrm{H})$, $7.78\left(\mathrm{~d}, 1 \mathrm{H}, J_{H, H}^{3} 8.1 \mathrm{~Hz}\right), 8.01\left(\mathrm{~d}, 1 \mathrm{H}, J_{H, H}^{3} 8.4 \mathrm{~Hz}, \mathrm{Napht}\right) .{ }^{13} \mathrm{C} \mathrm{NMR}\left(100.61 \mathrm{MHz}, \mathrm{CDCl}_{3}\right): \delta$ $27.3\left(\mathrm{CH}_{2}-\underline{\mathrm{CH}_{2}}-\mathrm{Napht}\right), 27.6\left(\underline{\mathrm{CH}}{ }_{2} \mathrm{C}=\mathrm{N}\right), 28.1\left(\mathrm{C}\left(\underline{\mathrm{CH}}_{3}\right)_{2}\right), 32.3\left(\underline{\mathrm{C}}\left(\mathrm{CH}_{3}\right)_{2}\right), 46.9\left(\underline{\mathrm{CH}}_{2} \mathrm{CH}_{2}-\right.$ Napht), $74.2\left(\mathrm{C}=\mathrm{N}-\underline{\mathrm{CH}}_{2}\right), 123.5,125.6,125.7,125.9,126.3,127.2,128.8,131.8,133.8,136.3$ (Napht), $151.3(\underline{\mathrm{C}}=\mathrm{N})$. Calcd. for $\mathrm{C}_{18} \mathrm{H}_{21} \mathrm{NO}: \mathrm{m} / z$ 267.1618 [M] $]^{+}$. Found: $m / z$ 267.1615 [M] ${ }^{+}$. Absorption maximum - $283 \mathrm{~nm}$, emission maximum - $337 \mathrm{~nm}$.

5-(2-(4-Methoxynaphthalen-1-yl)ethyl)-3,3-dimethyl-3,4-dihydro-2H-pyrrole 17b. Yellow oil, yield $0.254 \mathrm{~g}(12 \%)$; IR ( $\left.v_{\max }, \mathrm{cm}^{-1}\right)$ : 2955, 2932, 2866, $1640(\mathrm{C}=\mathrm{N}), 1588,1464,1391$, 1274, 1246, 1094, 766. ${ }^{1} \mathrm{H}$ NMR (400.13 MHz, $\left.\mathrm{CDCl}_{3}\right): \delta 1.05\left(\mathrm{~s}, 6 \mathrm{H}, \mathrm{C}\left(\mathrm{CH}_{3}\right)_{2}\right), 2.30(\mathrm{t}, 2 \mathrm{H}$, $\left.J_{H, H}^{4} 1.5 \mathrm{~Hz},\left(\mathrm{CH}_{3}\right)_{2} \mathrm{CCH}_{2} \mathrm{C}=\mathrm{N}\right), 2.69-2.74\left(\mathrm{~m}, 2 \mathrm{H}, \mathrm{CH}_{2}-\mathrm{CH}_{2}-\operatorname{arom}\right), 3.24-3.29$ (m, 2H, $\mathrm{CH}_{2} \underline{\mathrm{CH}}_{2}-$ arom), 3.58 (q, 2H, $J_{H, H}^{4} 1.5 \mathrm{~Hz},\left(\mathrm{CH}_{3}\right)_{2} \mathrm{C}-\mathrm{C}_{2}-\mathrm{N}=\mathrm{C}$ ), 3.93 (s, 3H, $\underline{\mathrm{H}}_{3} \mathrm{O}$ ), 6.69 (d, 
$\left.1 \mathrm{H}, J_{H, H}^{3} 7.9 \mathrm{~Hz}\right), 7.22\left(\mathrm{~d}, 1 \mathrm{H}, J_{H, H}^{3} 7.9 \mathrm{~Hz}\right), 7.43-7.47(\mathrm{~m}, 1 \mathrm{H}), 7.49-7.54(\mathrm{~m}, 1 \mathrm{H}), 7.96(\mathrm{~d}$, $\left.1 \mathrm{H}, J_{H, H}^{3} 8.4 \mathrm{~Hz}\right), 8.28-8.31\left(\mathrm{~m}, 1 \mathrm{H}\right.$, arom.). ${ }^{13} \mathrm{C} \mathrm{NMR}\left(100.61 \mathrm{MHz}, \mathrm{CDCl}_{3}\right): \delta 27.9$ $\left(\mathrm{C}\left(\underline{\mathrm{CH}}_{3}\right)_{2}\right), 29.1\left(\underline{\mathrm{CH}}_{2} \mathrm{C}=\mathrm{N}\right), 34.8\left(\mathrm{CH}_{2} \underline{\mathrm{CH}}_{2}-\mathrm{arom}\right), 38.1\left(\underline{\mathrm{C}}\left(\mathrm{CH}_{3}\right)_{2}\right), 52.6\left(\underline{\mathrm{CH}}_{2} \mathrm{CH}_{2}-\right.$ arom $), 55.4$ $\left(\underline{\mathrm{CH}}_{3} \mathrm{O}\right), 72.9\left(\underline{\mathrm{CH}}_{2}-\mathrm{N}=\mathrm{C}\right), 103.4,122.7,123.4,124.9,125.6,126.0,126.5,129.0,132.5,154.4$ (arom), $179.4(\underline{\mathrm{C}}=\mathrm{N})$. Calcd. for $\mathrm{C}_{19} \mathrm{H}_{23} \mathrm{NO}: m / z$ 281.1774 [M] ${ }^{+}$. Found: $m / z$ 281.1772 [M] $]^{+}$

5-(2-(4-Methoxynaphthalen-1-yl)ethyl)-3,3-dimethyl-3,4-dihydro-2H-pyrrole 1-oxide $18 b$. Pale yellow oil, yield $1.86 \mathrm{~g}(83 \%)$; IR $\left(v_{\max }, \mathrm{cm}^{-1}\right): 2959,1708(\mathrm{C}=\mathrm{N}), 1588,1464,1393,1373$, 1273, 1248, 1226, 1161, 1093, 767. ${ }^{1} \mathrm{H}$ NMR (400.13 MHz, $\left.\mathrm{CDCl}_{3}\right): \delta 0.93\left(\mathrm{~s}, 6 \mathrm{H}, \mathrm{C}\left(\mathrm{CH}_{3}\right)_{2}\right)$, 2.08 (s $\left.2 \mathrm{H},\left(\mathrm{CH}_{3}\right)_{2} \mathrm{CC}_{2} \mathrm{C}=\mathrm{N}\right), 2.79$ (t, $\left.2 \mathrm{H}, J_{H, H}^{3} 7.6 \mathrm{~Hz}, \underline{\mathrm{C}}_{2}-\mathrm{CH}_{2}-\mathrm{arom}\right), 3.19$ (t, $2 \mathrm{H}, J_{H, H}^{3} 7.6$ $\mathrm{Hz}, \mathrm{CH}_{2}-\underline{\mathrm{CH}}_{2}-$ arom), 3.66 (s, $\left.2 \mathrm{H},\left(\mathrm{CH}_{3}\right)_{2} \mathrm{C}-\mathrm{C}_{2}-\mathrm{N}=\mathrm{C}\right), 3.86\left(\mathrm{~s}, 3 \mathrm{H}, \mathrm{CH}_{3} \mathrm{O}\right), 6.63$ (d, $1 \mathrm{H}, J^{3}{ }_{H, H}$ $7.8 \mathrm{~Hz}), 7.15\left(\mathrm{~d}, 1 \mathrm{H}, J_{H, H}^{3} 7.8 \mathrm{~Hz}\right), 7.36-7.41(\mathrm{~m}, 1 \mathrm{H}), 7.44-7.49(\mathrm{~m}, 1 \mathrm{H}), 7.94\left(\mathrm{~d}, 1 \mathrm{H}, J_{H, H}^{3}\right.$ $8.5 \mathrm{~Hz}$ ), 8.20 - 8.24 (m, 1H, arom.). $\left.{ }^{13} \mathrm{C} \mathrm{NMR} \mathrm{(100.61} \mathrm{MHz,} \mathrm{CDCl}_{3}\right): \delta 26.8\left(\mathrm{CH}_{2}-\mathrm{C}_{2}-\right.$ arom), $27.7\left(\underline{\mathrm{CH}}_{2} \mathrm{C}=\mathrm{N}\right), 27.9\left(\mathrm{C}\left(\underline{\mathrm{CH}}_{3}\right)_{2}\right), 32.2\left(\underline{\mathrm{C}}\left(\mathrm{CH}_{3}\right)_{2}\right), 46.9\left(\mathrm{C}_{2} \mathrm{CH}_{2}-\operatorname{arom}\right), 55.3\left(\underline{\mathrm{CH}}_{3} \mathrm{O}\right), 74.3$ $\left(\mathrm{C}=\mathrm{N}-\underline{\mathrm{CH}}_{2}\right), 103.2,122.5,123.2,124.8,125.6,125.7,126.5,128.0,132.4$ (arom), $149.8(\underline{\mathrm{C}}=\mathrm{N})$, 154.4 (arom). Calcd. for $\mathrm{C}_{19} \mathrm{H}_{23} \mathrm{NO}_{2}$ : $m / z, 297.1773[\mathrm{M}]^{+}$. Found: $m / z$ 297.1772 [M] $]^{+}$. Absorption maximum $-302 \mathrm{~nm}$, emission maximum $-357 \mathrm{~nm}$.

2,4,4-Trimethyl-2-(2-(naphthalen-1-yl)ethyl)-3,4-dihydro-2H-pyrrole 1-oxide 19a. To a stirred solution of methylmagnesium iodide, prepared from magnesium $(0.510 \mathrm{~g}, 21.2 \mathrm{mmol})$ and methyl iodide $(1.31 \mathrm{~mL}, 21.0 \mathrm{mmol})$ in anhydrous ether $(15 \mathrm{~mL})$, a solution of $18 \mathbf{a}(1.4 \mathrm{~g}, 5.24$ $\mathrm{mmol})$ in anhydrous ether $(5 \mathrm{~mL})$ was added. Reaction mixture was stirred for 2 hours at room temperature, the excess of Grignard reagent was decomposed by addition of saturated ammonium chloride solution $(15 \mathrm{~mL})$. Ether solution was separated and water layer was extracted with ether $(4 \times 20 \mathrm{~mL})$. Combined organic extract were dried with $\mathrm{MgSO}_{4}$, the solvent was removed under reduced pressure. The residue was dissolved in chloroform (30 $\mathrm{mL})$, manganese dioxide $(0.2 \mathrm{~g})$ was added and the resulting mixture was stirred for 1 hour at room temperature. Then manganese dioxide was filtered off and washed with chloroform (40 mL). The filtrate was concentrated in vacuo, compound 19a was purified by chromatography with a mixture of chloroform - methanol (50:1) as eluent. Colorless crystals, yield $0.9 \mathrm{~g}(61 \%)$; mp 111 - $112{ }^{\circ} \mathrm{C}$ (from mixture hexane - ethylacetate 5:1); IR $\left(v_{\max }, \mathrm{cm}^{-1}\right): 2963,1577(\mathrm{C}=\mathrm{N}), 1459$, 1264, 1237, 1170, 800, 781, 729. ${ }^{1} \mathrm{H}$ NMR (400.13 $\left.\mathrm{MHz}, \mathrm{CDCl}_{3}\right): \delta 1.26\left(\mathrm{~s}, 3 \mathrm{H}, \mathrm{C}\left(\mathrm{C}_{3}\right)_{2}\right), 1.28$ (s, 3H, C( $\left.\left.\underline{\mathrm{CH}}_{3}\right)_{2}\right), 1.54$ (s, 3H, $\left.\underline{\mathrm{CH}}_{3}\right), 1.92-2.00$ (m, 2H, $\left.\underline{\mathrm{C}}_{2} \mathrm{CH}_{2}-\mathrm{Napht}\right), 2.24-2.30$ (m, 2H, $\left.\mathrm{C}_{2} \mathrm{CH}_{2}-\mathrm{Napht}\right), 3.01-3.06\left(\mathrm{~m}, 2 \mathrm{H},\left(\mathrm{CH}_{3}\right)_{2} \mathrm{CC}_{2}\right), 6.73(\mathrm{~s}, 1 \mathrm{H}, \mathrm{C} \underline{H}=\mathrm{N}), 7.30-7.37$ (m, 2H), $7.42-7.46(\mathrm{~m}, 1 \mathrm{H}), 7.49-7.53(\mathrm{~m}, 1 \mathrm{H}),, 7.68\left(\mathrm{~d}, 1 \mathrm{H}, J_{H, H}^{3} 7.8 \mathrm{~Hz}\right), 7.79-7.82(\mathrm{~m}, 1 \mathrm{H}), 8.10$ (d, $1 \mathrm{H}, J_{H, H}^{3} 8.5 \mathrm{~Hz}$, Napht). ${ }^{13} \mathrm{C} \mathrm{NMR}\left(100.61 \mathrm{MHz}, \mathrm{CDCl}_{3}\right): \delta 27.1\left(\mathrm{CH}_{2}-\mathrm{CH}_{2}\right.$-arom), 27.7 $\left.\left(\underline{\mathrm{CH}_{2} \mathrm{C}}\left(\mathrm{CH}_{3}\right)_{2}\right), 28.6\left(\mathrm{C}\left(\underline{\mathrm{CH}}_{3}\right)_{2}\right), 29.1\left(\mathrm{C}_{(\mathrm{CH}}\right)_{2}\right), 38.1\left(\underline{\mathrm{C}}\left(\mathrm{CH}_{3}\right)_{2}\right), 39.8\left(\underline{\mathrm{CH}}_{3}\right), 45.8\left(\underline{\mathrm{CH}}_{2}-\mathrm{CH}_{2}-\right.$ Napht), $77.3\left(\mathrm{C}=\mathrm{N}-\underline{\mathrm{C}}\left(\mathrm{CH}_{3}\right) \mathrm{CH}_{2}\right), 123.8,125.6$ (2C), 126.1, 126.2, 127.0, 128.8, 131.9, 133.9, 137.4 (Napht), $141.2(\underline{\mathrm{CH}}=\mathrm{N})$. Calcd. for $\mathrm{C}_{18} \mathrm{H}_{21} \mathrm{NO}: \mathrm{m} / z$ 281.1774 [M] ${ }^{+}$. Found: $m / z$ 281.1771 $[\mathrm{M}]^{+}$. Absorption maximum $-284 \mathrm{~nm}$, emission maximum $-337 \mathrm{~nm}$.

2-(2-(4-Methoxynaphthalen-1-yl)ethyl)-2,4,4-trimethyl-3,4-dihydro-2H-pyrrole 1-oxide 19b was synthesized according to the procedure described for 19a from $\mathbf{1 8 b}(1.56 \mathrm{~g}, 5.24 \mathrm{mmol})$. 
Colorless crystals, yield $1.21 \mathrm{~g}$ (74\%); mp $144-146^{\circ} \mathrm{C}$ (from mixture hexane - ethyl acetate 4:1); IR $\left(v_{\max }, \mathrm{cm}^{-1}\right): 2965,2927,1589(\mathrm{C}=\mathrm{N}), 1464,1393,1268,1250,1161,1090,822,775 .{ }^{1} \mathrm{H}$ NMR (300.13 MHz, $\left.\mathrm{CDCl}_{3}\right): \delta 1.26$ (s, 3H, C $\left.\left(\mathrm{C}_{3}\right)_{2}\right), 1.28$ (s, $\left.3 \mathrm{H}, \mathrm{C}\left(\mathrm{C}_{3}\right)_{2}\right), 1.53$ (s, $\left.3 \mathrm{H}, \underline{\mathrm{C}}_{3}\right)$, $1.93-2.02$ (m, $2 \mathrm{H}, \mathrm{C}_{2} \mathrm{CH}_{2}$-arom), $2.21-2.29$ (m, 2H, $\mathrm{CH}_{2} \mathrm{CH}_{2}$-arom), $2.92-2.99$ (m, $2 \mathrm{H}$, $\left.\left(\mathrm{CH}_{3}\right)_{2} \mathrm{CC}_{2}\right), 3.94$ (s, $\left.3 \mathrm{H}, \mathrm{C}_{3} \mathrm{O}\right), 6.69\left(\mathrm{~d}, 1 \mathrm{H}, J_{H, H}^{3} 7.8 \mathrm{~Hz}\right.$, arom.), 6.79 (s, $\left.1 \mathrm{H}, \mathrm{C} \underline{\mathrm{H}}=\mathrm{N}\right), 7.21$ $\left(\mathrm{d}, 1 \mathrm{H}, J_{H, H}^{3} 7.8 \mathrm{~Hz}\right), 7.41-7.47(\mathrm{~m}, 1 \mathrm{H}), 7.50-7.56(\mathrm{~m}, 1 \mathrm{H}), 8.00\left(\mathrm{~d}, 1 \mathrm{H}, J_{H, H}^{3} 8.3 \mathrm{~Hz}\right), 8.25-$ $8.29\left(\mathrm{~m}, 1 \mathrm{H}\right.$, arom). ${ }^{13} \mathrm{C}$ NMR $\left(75.46 \mathrm{MHz}, \mathrm{CDCl}_{3}\right): \delta 27.0\left(\mathrm{CH}_{2}-\mathrm{CH}_{2}\right.$-arom), 27.2 $\left(\underline{\mathrm{CH}}_{2} \mathrm{C}\left(\mathrm{CH}_{3}\right)_{2}\right), 28.5\left(\mathrm{C}\left(\underline{\mathrm{C}} \mathrm{H}_{3}\right)_{2}\right), 29.0\left(\mathrm{C}\left(\underline{\mathrm{CH}}_{3}\right)_{2}\right), 38.3\left(\underline{\mathrm{C}}\left(\mathrm{CH}_{3}\right)_{2}\right), 39.9\left(\underline{\mathrm{CH}}_{3}\right), 45.8\left(\underline{\mathrm{CH}}_{2}-\mathrm{CH}_{2}-\right.$ arom $), 55.5\left(\mathrm{CH}_{3} \mathrm{O}\right), 77.5\left(\mathrm{C}=\mathrm{N}-\underline{\mathrm{C}}\left(\mathrm{CH}_{3}\right) \mathrm{CH}_{2}\right), 103.5,122.6,123.6,125.0,125.8,126.0,126.7$, 129.2, 132.6 (arom), $142.1(\underline{\mathrm{CH}}=\mathrm{N}), 154.4$ (arom.). Calcd. for $\mathrm{C}_{20} \mathrm{H}_{25} \mathrm{NO}_{2}$ : $\mathrm{m} / z 311.1876[\mathrm{M}]^{+}$. Found: $m / z 311.1889[\mathrm{M}]^{+}$. Absorption maximum $-301 \mathrm{~nm}$, emission maximum $-358 \mathrm{~nm}$.

\section{References}

1. Tsai P.; Elas M.; Parasca A. D.; Barth E. D.; Mailer C.; Halpern H. J.; Rosen G. M., J. Chem. Soc., Perkin Trans. 2 2001, 6, 875. http://dx.doi.org/10.1039/b101945g

2. Gosset G.; Clement J. L.; Culcasi M.; Rockenbauer A.; Pietri S. Bioorg. Med. Chem. 2011, 19, 2218.

http://dx.doi.org/10.1016/j.bmc.2011.02.040

PMid:21420303

3. Kim S. U.; Liu Y.; Nash K. M.; Zweier J. L.; Rockenbauer A.; Villamena F. A. J. Am. Chem. Soc. 2010, 132, 17157.

http://dx.doi.org/10.1021/ja105198c PMid:21070040 PMCid:PMC3121924

4. Villamena F. A.; Xia S.; Merle J. K.; Lauricella R.; Tuccio B.; Hadad C. M.; Zweirer J. L. J. Am. Chem. Soc. 2007, 129, 8177.

http://dx.doi.org/10.1021/ja0702622

PMid:17564447 PMCid:PMC2527741

5. Blough, N.V.; Simpson, D.J. J. Am. Chem. Soc. 1988, 110, 1915. http://dx.doi.org/10.1021/ja00214a041

6. Pou S.; Bhan A.; Bhadti V. S.; Wu S. Y.; Hosmane R. S.; Rosen G. M. The FASEB J. 1995, 9, 1085.

PMid:7649408

7. Hauck S.; Lorat Y.; Leinich F.; Trommer W. E. Appl. Magn. Reson. 2009, 36, 133. http://dx.doi.org/10.1007/s00723-009-0025-3

8. Janzen E. G.; Zhang Y.-K.; Haire D. L. J. Am. Chem. Soc. 1994, 116, 3738. http://dx.doi.org/10.1021/ja00088a009 
9. Bonnett R.; Brown R. F. C.; Clark V. M.; Sutherland I. O. and Todd A. J. Chem. Soc. 1959, 2094.

10. Patel A.; Rohr-Udilova N.; Rosenau T.; Stolze K. Bioorg. Med. Chem. 2011, 19, 7643. http://dx.doi.org/10.1016/j.bmc.2011.10.017 PMid:22094277

11. Kornblum N.; Blackwood R. K.; Powers J. W. J. Am. Chem. Soc. 1957, 79, 2507. http://dx.doi.org/10.1021/ja01567a041

12. Tsai, P.; Ichikawa, K.; Mailer, C.; Pou, S.; Halpern, H. J.; Robinson, B. H.; Nielsen, R.; Rosen, G. M. J. Org. Chem. 2003, 68, 7811. http://dx.doi.org/10.1021/jo0350413 PMid:14510560

13. Keana, J. F. W. Chem. Rev. 1978, 78, 37. http://dx.doi.org/10.1021/cr60311a004

14. Morozov, D. A.; Kirilyuk, I. A.; Komarov, D. A.; Goti, A.; Bagryanskaya, I. Y.; Kuratieva, N. V.; Grigor'ev, I. A. J. Org. Chem. 2012, 77, 10688.

http://dx.doi.org/10.1021/jo3019158

PMid:23130653

15. Nazarski, R. B.; Skowronski, R. J. Chem. Soc., Perkin Trans. 1 1989, 1603.

16. Bapat, J. B.; Black, D. St. C. Austr. J. Chem. 1968, 21, 2521. http://dx.doi.org/10.1071/CH9682521

17. G.M. Sheldrick, SHELX-97 - Programs for Crystal Structure Analysis, Release 97-2, University of Goetingen, Goettingen, Germany.

18. A.L. Spek, PLATON, A Multiperpose Crystallographic Tool, Version 10M, Utrecht University, Utrecht, The Netherlands, 2003; A.L. Spek, J. Appl. Crystallogr. 2003, 36, 7. http://dx.doi.org/10.1107/S0021889802022112

19. Meng Q.; Sun Y.; Ratovelomanana - Vidal V.; Genete J. P.; Zhang Z. J. Org. Chem. 2008, 73,3842 .

http://dx.doi.org/10.1021/jo800228e

PMid:18429633

20. Wislicenus, W.; Wren, H. Ber. Deutsch. Chem. Ges. 1905, 38, 507.

21. Allen F.H.; Kennard O.; Watson D.G. et al. J. Chem. Soc. Perkin Trans. II 1987, 12, 1. http://dx.doi.org/10.1039/p298700000s1

22. Rowland R. S.; Taylor R. J. Phys. Chem. 1996, 100, 7384.

http://dx.doi.org/10.1021/jp953141+

23. Cambridge Structural Database (Version 5.27), University of Cambridge, UK; F. H. Allen, Acta Crystallogr., Sect. B 2002, 58, 380.

PMid:12037359 